\title{
A POSTERIORI ERROR ESTIMATES FOR A NONCONFORMING FINITE ELEMENT DISCRETIZATION OF THE HEAT EQUATION
}

\author{
SERGe NicAise ${ }^{1}$ AND NAdir Soualem ${ }^{1}$
}

\begin{abstract}
The paper presents an a posteriori error estimator for a (piecewise linear) nonconforming finite element approximation of the heat equation in $\mathbb{R}^{d}, d=2$ or 3 , using backward Euler's scheme. For this discretization, we derive a residual indicator, which use a spatial residual indicator based on the jumps of normal and tangential derivatives of the nonconforming approximation and a time residual indicator based on the jump of broken gradients at each time step. Lower and upper bounds form the main results with minimal assumptions on the mesh. Numerical experiments and a space-time adaptive algorithm confirm the theoretical predictions.
\end{abstract}

Mathematics Subject Classification. 65N15, 65N30, 65M50.

Received: July 12, 2004.

\section{INTRODUCTION}

This paper deals with the a posteriori analysis of the heat equation approximated using backward Euler's scheme in time and a (piecewise linear) nonconforming finite element approximation in space. There are several reasons to use nonconforming approximations. For example the approximation of the Stokes system requires the stability of the method, namely the discrete space has to satisfy the so-called inf-sup condition with a constant independent of the aspect ratio of the elements. Unfortunately standard conforming elements (like the mini element, the Taylor-Hood element, etc.) are not stable on anisotropic meshes (meshes for which the aspect ratio is no more bounded [3] and often used for the approximation of edge singularities and/or boundary layers), see $[2,4]$ and the references cited there. Therefore the use of nonconforming elements may be recommended since they are unconditionally stable [5].

As a first attempt we consider the case of the heat equation approximated by a piecewise linear nonconforming finite element space based on a regular family of triangulations. However our method may be extended to the Stokes system and to the use of anisotropic meshes. This will be investigated in forthcoming works.

In the conforming case several approaches have been introduced to define error estimators for the heat equation and the Stokes system $[6-9,17,18,20,22]$. To be able to extend these techniques to nonconforming spatial approximations, as for elliptic problems [14], we need to be able to estimate the consistency term appearing in the error equation. As in [14], this term is managed using a Helmholtz decomposition of the error. This allows us to extend the results from $[7-9,22]$ to the nonconforming case.

\footnotetext{
Keywords and phrases. Error estimator, nonconforming FEM, heat equation.

1 Université de Valenciennes et du Hainaut Cambrésis, MACS, ISTV, 59313 Valenciennes Cedex 9, France.

Serge.Nicaise@univ-valenciennes.fr; Nadir.Soualem@univ-valenciennes.fr
} 
The schedule of the paper is the following one: Section 2 recalls the continuous and its discretizations. In Section 3 we give some analytical tools, in particular some properties satisfied by the spatial error and its Helmholtz like decomposition. Section 4 is devoted to the a posteriori analysis of the time discretization. The efficiency and reliability of the spatial error estimator are established in Section 5. The a posteriori analysis of the full discrete problem is considered in Section 6, where we show the efficiency and reliability of the sum of the spatial and time error estimators. Finally in Section 7, we present some numerical tests which confirm our theoretical analysis. We further describe a space-time adaptive algorithm, which is validated by two relevant examples.

\section{The COntinuous, time SEMI-Discrete AND FUll Discrete PROBlems}

Let $\Omega$ be an open bounded of $\mathbb{R}^{d}, d=2$ or 3 , with a polygonal $(d=2)$ or polyhedral $(d=3)$ boundary $\Gamma$. For the sake of simplicity, we assume that $\Omega$ is simply connected and that its boundary is connected. Let $T$ be a positive and fixed real number.

Let us introduce some notation used in the whole paper: for shortness, if $D$ is a subset of $\Omega$, the $L^{2}(D)$-norm (resp. $L^{2}(D)$ inner product) will be denoted by $\|\cdot\|_{D}$ (resp. $\left.(\cdot, \cdot)_{D}\right)$. In the case $D=\Omega$, we will drop the index $\Omega$. The usual norm and seminorm of the standard Sobolev space $H^{s}(D)$, with $s>0$, are denoted by $\|\cdot\|_{s, D}$ and $|\cdot|_{s, D}$.

In this paper we consider the following heat equation: Let $u$ be the solution of

$$
\left\{\begin{array}{cc}
\frac{\partial u}{\partial t}-\Delta u=f & \text { in } \Omega \times] 0, T[ \\
u(., t)=0 & \text { on } \Gamma \times] 0, T[ \\
u(., 0)=u_{0} & \text { in } \Omega .
\end{array}\right.
$$

The datum $f$ is supposed to satisfy $f \in L^{2}\left(0, T ; H^{-1}(\Omega)\right)$ and the initial value $u_{0} \in L^{2}(\Omega)$. Under these assumptions, problem (1) or equivalently

$$
\left(\partial_{t} u(t), v\right)+(\nabla u(t), \nabla v)=(f(t), v), \forall v \in H_{0}^{1}(\Omega), \forall \text { a.e. } t \in(0, T),
$$

has a unique (weak) solution in $L^{2}\left(0, T ; H_{0}^{1}(\Omega)\right) \cap C\left([0, T] ; L^{2}(\Omega)\right)$.

\subsection{Time discretization using Euler's scheme}

We now suppose that $f \in C\left([0, T] ; H^{-1}(\Omega)\right)$. We further introduce a partition of $[0, T]$ into subintervals $\left[t_{p-1}, t_{p}\right], 1 \leq p \leq N$ such that $0=t_{0}<t_{1}<\cdots<t_{N}=T$. Denote by $\tau_{p}=t_{p}-t_{p-1}$ the length of $\left[t_{p-1}, t_{p}\right]$ and by $\tau=\max _{p} \tau_{p}$ the global time mesh size.

The semi-discrete approximation of the continuous problem (1) by a backward Euler scheme consists in finding a sequence $\left(u^{p}\right)_{0 \leq p \leq N}$ solution of

$$
\begin{cases}\frac{u^{p}-u^{p-1}}{\tau_{p}}-\Delta u^{p}=f^{p} & \text { in } \Omega \quad 1 \leq p \leq N, \\ u^{p}=0 & \text { on } \Gamma \quad 1 \leq p \leq N, \\ u^{0}=u_{0} & \text { in } \Omega,\end{cases}
$$

with $f^{p}=f\left(\cdot, t_{p}\right)$. This problem admits a unique weak solution $u^{p} \in H_{0}^{1}(\Omega)$, whose variational formulation is

$$
\int_{\Omega} u^{p} v+\tau_{p} \int_{\Omega} \nabla u^{p} \cdot \nabla v=\int_{\Omega} u^{p-1} v+\tau_{p} \int_{\Omega} f^{p} v \quad \forall v \in H_{0}^{1}(\Omega) .
$$

The unique solvability of the variational formulation (4) is then a direct consequence of the Lax-Milgram lemma. 


\subsection{Full discretization}

Problem (4) is now discretized by a nonconforming finite element method. For that purpose, for all $p=$ $0,1, \cdots, N$, let us fix a conforming mesh $T_{p h}$ of $\Omega$ which form a regular family of triangulations in Ciarlet's sense ([11], p. 124), i.e., there exists $\sigma>0$ such that

$$
h_{K} / \rho_{K} \leq \sigma, \forall K \in T_{p h}
$$

where we recall that $h_{K}$ is the diameter of $K$ and $\rho_{K}$ is the diameter of the largest ball included into $K$. All elements are triangles or tetrahedra and will be denoted by $K$. For all $p$, we denote by $h_{p}=\max _{K \in T_{p h}} h_{K}$. The set of all edges/faces of $T_{p h}$ is denoted by $\mathcal{E}_{p h}$. Let $\mathcal{E}_{p h}^{i n t}$ be the set of interior edges/faces of $T_{p h}$ and $\mathcal{E}_{K}$ be the set of the edges/faces of the element $K$. Finally for an edge/face $E \in \mathcal{E}_{K} \cap \mathcal{E}_{L}$ we denote by $h_{E}=\frac{1}{2}\left(\frac{d|K|}{|E|}+\frac{d|L|}{|E|}\right)$, its mean height.

Introduce the Crouzeix-Raviart nonconforming finite element space:

$$
\begin{aligned}
X_{p h}^{0}= & \left\{v \in L^{2}(\Omega): v_{\mid K} \in \mathbb{P}_{1}, \forall K \in T_{p h},\right. \\
& \int_{E} v_{\mid K}=\int_{E} v_{\mid L}, \forall E \in \mathcal{E}_{K} \cap \mathcal{E}_{L} \cap \mathcal{E}_{p h}^{i n t}, K, L \in T_{p h}, \\
& \left.\int_{E} v_{\mid K}=0, \forall E \in \mathcal{E}_{K} \cap \Gamma, K \in T_{p h}\right\} .
\end{aligned}
$$

The full discrete approximation of problem (1) using Euler's scheme and the Crouzeix-Raviart nonconforming finite element, is then given by: given an approximation $u_{h}^{0} \in X_{0 h}^{0}$ of $u_{0}$, find $u_{h}^{p} \in X_{p h}^{0}, 1 \leq p \leq N$, such that:

$$
\int_{\Omega} u_{h}^{p} v_{h}+\tau_{p} \sum_{K \in T_{p h}} \int_{K} \nabla u_{h}^{p} \nabla v_{h}=\int_{\Omega} u_{h}^{p-1} v_{h}+\tau_{p} \int_{\Omega} f^{p} v_{h}
$$

for all $v_{h} \in X_{p h}^{0}$.

Note that the Crouzeix-Raviart elements were recently used in [1] for the discretization of a mixed formulation of the Laplace equation and that the nonconformity of the approximation also renders their a posteriori analysis more delicate.

Definition 2.1. Let $u^{p}$ be a solution of (4) and $u_{h}^{p}$ a solution of (5), then we denote the spatial error by

$$
e^{p}=u^{p}-u_{h}^{p}
$$

Let us finish this section by introducing some useful notation and properties used below.

The notation $a \lesssim b$ and $a \sim b$ means the existence of positive constants $C_{1}$ and $C_{2}$ (which are independent of the mesh size of the triangulations, of the time step size and of the function under consideration) such that $a \leq C_{2} b$ and $C_{1} b \leq a \leq C_{2} b$, respectively.

For a boundary edge/face $E$ we denote the outward normal vector by $n_{E}$. In $2 \mathrm{D}$, we further define the tangent vector by $t_{E}=\left(-n_{E 2}, n_{E 1}\right)$ if $n_{E}=\left(n_{E 1}, n_{E 2}\right)$. Given an interior edge/face $E$, we choose an arbitrary normal direction $n_{E}$ and denote by $K_{i n}$ and $K_{\text {ext }}$ the two elements sharing this edge/face. Without any restriction, we may suppose here that $n_{E}$ is pointing to $K_{e x t}$ like in Figure 1. In 2D, denote as before the tangent vector by $t_{E}=\left(-n_{E 2}, n_{E 1}\right)$ if $n_{E}=\left(n_{E 1}, n_{E 2}\right)$.

For the analysis of the nonconforming approximation, we will use the following Crouzeix-Raviart property:

$$
\int_{E} \llbracket \llbracket u_{h} \rrbracket_{E}=0 \quad \forall E \in \mathcal{E}_{p h}, \forall u_{h} \in X_{p h}^{0},
$$




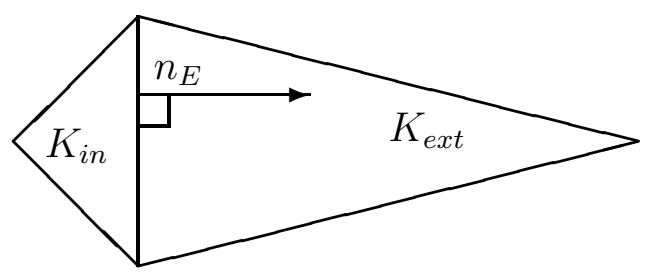

Figure 1. Two elements sharing the edge $E$.

where the jump of some function $v$ across an edge/face $E$ at a point $x$ is defined by

$$
\llbracket v(x) \rrbracket_{E}= \begin{cases}\lim _{\alpha \rightarrow 0^{+}} v\left(x+\alpha n_{E}\right)-v\left(x-\alpha n_{E}\right) & \text { if } E \in \mathcal{E}_{p h}^{i n t}, \\ v(x) & \text { if } E \in \mathcal{E}_{p h} \backslash \mathcal{E}_{p h}^{i n t} .\end{cases}
$$

Note that the sign of $\llbracket v(x) \rrbracket_{E}$ depends on the orientation of $n_{E}$. However, quantity like a gradient jump $\llbracket \nabla v \cdot n_{E} \rrbracket_{E}$ is independent of this orientation.

For a function $v \in X_{p h}^{0}$ we define its broken gradient $\nabla_{h} v$ by

$$
\left(\nabla_{h} v\right)_{\mid K}=\nabla\left(v_{\mid K}\right), \forall K \in T_{p h} .
$$

In the sequel we will use local patches: for an element $K$ we define $\omega_{K}$ as the union of all elements having a common edge/face with $K$, for an edge/face $E$, let $\omega_{E}$ be the union of both elements having $E$ as edge/face and finally for a node $x$, let $\omega_{x}$ be the union of both elements having $x$ as node. Similarly denote by $\tilde{\omega}_{K}$ (resp. $\tilde{\omega}_{E}$ ) the union of all triangles sharing a node with $K$ (resp. E).

We further need the standard $\mathbb{P}_{1}$ conforming finite element spaces

$$
\begin{aligned}
V_{p h} & =\left\{v \in H^{1}(\Omega): v_{\mid K} \in \mathbb{P}_{1}, \forall K \in T_{p h}\right\}, \\
V_{p h}^{0} & =V_{p h} \cap H_{0}^{1}(\Omega) .
\end{aligned}
$$

For our error analysis we require an interpolant that maps $X_{p h}^{0} \oplus H_{0}^{1}(\Omega)$ to $V_{p h}^{0}$. Hence Lagrange interpolation operator is unsuitable but Clément like interpolation operator is more appropriate. To write the results in the largest setting as possible, let us set

$$
\begin{aligned}
Y_{p h}= & \left\{v \in L^{2}(\Omega): v_{\mid K} \in H^{1}(K), \forall K \in T_{p h},\right. \\
& \left.\int_{E} v_{\mid K}=\int_{E} v_{\mid L}, \forall E \in \mathcal{E}_{K} \cap \mathcal{E}_{L} \cap \mathcal{E}_{p h}^{i n t}, K, L \in T_{p h},\right\}, \\
Y_{p h}^{0}= & \left\{v \in L^{2}(\Omega): v_{\mid K} \in H^{1}(K), \forall K \in T_{p h},\right. \\
& \int_{E} v_{\mid K}=\int_{E} v_{\mid L}, \forall E \in \mathcal{E}_{K} \cap \mathcal{E}_{L} \cap \mathcal{E}_{p h}^{i n t}, K, L \in T_{p h}, \\
& \left.\int_{E} v_{\mid K}=0, \forall E \in \mathcal{E}_{K} \cap \Gamma, K \in T_{p h}\right\} .
\end{aligned}
$$

Note that $H^{1}(\Omega) \subset Y_{p h}$ and that $X_{p h}^{0} \oplus H_{0}^{1}(\Omega) \subset Y_{p h}^{0}$.

Recall that the Clément interpolation operator is defined as follows: Denote by $\mathcal{N}_{p h}$ the set of nodes of the triangulation $T_{p h}$ and by $\mathcal{N}_{p h}^{i n t}$ the set of interior nodes of the triangulation $T_{p h}$. For each node $x$ denote further by $\lambda_{x}$ the standard hat function associated with $x$, namely $\lambda_{x} \in V_{p h}$ and satisfies

$$
\lambda_{x}(y)=\delta_{x, y}, \forall y \in \mathcal{N}_{p h} .
$$


For any $v \in Y_{p h}$ and $w \in Y_{p h}^{0}$, we define $I_{C} v$ and $I_{C}^{0} w$ by

$$
\begin{aligned}
& I_{C} v=\sum_{x \in \mathcal{N}_{p h}}\left|\omega_{x}\right|^{-1}\left(\int_{\omega_{x}} v\right) \lambda_{x}, \\
& I_{C}^{0} w=\sum_{x \in \mathcal{N}_{p h}^{i n t}}\left|\omega_{x}\right|^{-1}\left(\int_{\omega_{x}} w\right) \lambda_{x} .
\end{aligned}
$$

Note that $I_{C} v$ belongs to $V_{p h}$, while $I_{C}^{0} w$ belongs to $V_{p h}^{0}$. Moreover these operators have the following properties:

Lemma 2.2. For all $v \in Y_{p h}$ and $w \in Y_{p h}^{0}$, we have

$$
\begin{aligned}
\left\|v-I_{C} v\right\|_{K} & \lesssim h_{K}\left\|\nabla_{h} v\right\|_{\tilde{\omega}_{K}}, \forall K \in T_{p h}, \\
\left\|v-I_{C} v\right\|_{E} & \lesssim h_{E}^{1 / 2}\left\|\nabla_{h} v\right\|_{\tilde{\omega}_{E}}, \forall E \in \mathcal{E}_{p h}, \\
\left\|w-I_{C}^{0} w\right\|_{K} & \lesssim h_{K}\left\|\nabla_{h} w\right\|_{\tilde{\omega}_{K}}, \forall K \in T_{p h}, \\
\left\|w-I_{C}^{0} w\right\|_{E} & \lesssim h_{E}^{1 / 2}\left\|\nabla_{h} w\right\|_{\tilde{\omega}_{E}}, \forall E \in \mathcal{E}_{p h}^{i n t}, \\
\left\|\nabla I_{C}^{0} w\right\|_{K} & \lesssim\left\|\nabla_{h} w\right\|_{\tilde{\omega}_{K}}, \forall K \in T_{p h} .
\end{aligned}
$$

Proof. For $v$ and $w$ in $H^{1}(\Omega)$, the above properties are proved in [12] (see also [19,21] for other interpolation operators) using scaling arguments, but a careful analysis of their proof reveals that these properties hold for $v$ and $w$ as in the statement of the Lemma.

The mean value of some function $v$ on an edge/face $E$ is defined by

$$
\mathcal{M}_{E}(v)=\frac{1}{|E|} \int_{E} v
$$

In the sequel we often need the following Green's formulas: if $D$ is a bounded open subset of $\mathbb{R}^{2}$ and $v, w \in$ $H^{1}(D)$, then we have

$$
\int_{D} \nabla v \cdot \operatorname{curl} w=\int_{\partial D} v \operatorname{curl} w \cdot n=\int_{\partial D} \nabla v \cdot t w
$$

where $t$ is the unit tangent vector along $\partial D$ and $\operatorname{curl} w$ is the vectorial curl of $w$, namely curl $w=\left(\begin{array}{c}\partial_{2} w \\ -\partial_{1} w\end{array}\right)$. Similarly if $D$ is a bounded open subset of $\mathbb{R}^{3}$ and $v \in H^{1}(D), w \in H^{1}(D)^{3}$ then we have

$$
\int_{D} \nabla v \cdot \operatorname{curl} w=\int_{\partial D} v \operatorname{curl} w \cdot n=\int_{\partial D}(\nabla v \times n) \cdot w .
$$

We finally introduce the gradient jump of $u_{h}^{p}$ in normal and tangential direction by

$$
J_{E, n}^{p}= \begin{cases}\llbracket \nabla u_{h}^{p} \cdot n_{E} \rrbracket_{E} & \text { if } E \in \mathcal{E}_{p h}^{i n t}, \\ 0 & \text { if } E \in \mathcal{E}_{p h} \backslash \mathcal{E}_{p h}^{i n t} .\end{cases}
$$

If $d=2$, then

If $d=3$, then

$$
J_{E, t}^{p}= \begin{cases}\llbracket \nabla u_{h}^{p} \cdot t_{E} \rrbracket_{E} & \text { if } E \in \mathcal{E}_{p h}^{i n t}, \\ -\nabla u_{h}^{p} \cdot t_{E} & \text { if } E \in \mathcal{E}_{p h} \backslash \mathcal{E}_{p h}^{i n t} .\end{cases}
$$

$$
J_{E, t}^{p}= \begin{cases}\llbracket \nabla u_{h}^{p} \times n_{E} \rrbracket_{E} & \text { if } E \in \mathcal{E}_{p h}^{i n t}, \\ -\nabla u_{h}^{p} \times n_{E} & \text { if } E \in \mathcal{E}_{p h} \backslash \mathcal{E}_{p h}^{i n t} .\end{cases}
$$




\section{Some Analytical tools}

In this section we collect different properties satisfied by the spatial error $e^{p}$ that we will use in the proof of the spatial error bounds.

Lemma 3.1 (Galerkin orthogonality). The error $e^{p}$ satisfies the Galerkin orthogonality relation

$$
\sum_{K \in T_{p h}} \int_{K} \nabla_{h} e^{p} \cdot \nabla v_{h}=\int_{\Omega} \frac{e^{p-1}-e^{p}}{\tau_{p}} v_{h}, \forall v_{h} \in V_{p h}^{0}
$$

Proof. It suffices to subtract (4) with $v=v_{h} \in V_{p h}^{0}$ to the identity (5).

Lemma 3.2. Let $\varphi \in H^{1}(\Omega)$ if $d=2$ and $\varphi \in H^{1}(\Omega)^{3}$ if $d=3$. Then the error satisfies the following identity

$$
\int_{\Omega} \nabla_{h} e^{p} \cdot \operatorname{curl} \varphi=\sum_{E \in \mathcal{E}_{p h}} \int_{E} J_{E, t}^{p} \cdot \varphi
$$

Proof. Assume that $d=2$. Integrations by parts in $\Omega$ and in each element $K$ give (see (14))

$$
\begin{aligned}
\int_{\Omega} \nabla_{h} e^{p} \cdot \operatorname{curl} \varphi & =\int_{\Omega} \nabla u^{p} \cdot \operatorname{curl} \varphi-\sum_{K \in T_{p h}} \int_{K} \nabla u_{h}^{p} \cdot \operatorname{curl} \varphi \\
& =\int_{\Gamma} \operatorname{curl} \varphi \cdot n u^{p}-\sum_{K \in T_{p h}} \int_{\partial K} \nabla u_{h}^{p} \cdot t_{K} \varphi .
\end{aligned}
$$

As $u^{p} \in H_{0}^{1}(\Omega)$ and $\varphi \in H^{1}(\Omega)$, we conclude using the definition of $J_{E, t}^{p}$.

The proof is similar in dimension 3 using (15).

Lemma 3.3 (error orthogonality). The error satisfies

$$
\sum_{K \in T_{p h}} \int_{K} \nabla_{h} e^{p} \cdot \operatorname{curl} \varphi_{h}=0, \forall \varphi_{h} \in V_{p h} \text { if } d=2 \text { and } \varphi_{h} \in\left(V_{p h}\right)^{3} \text { if } d=3 .
$$

Proof. Consider an arbitrary element $\varphi_{h}$ in $V_{p h}$ if $d=2$ or in $\left(V_{p h}\right)^{3}$ if $d=3$. As before, by integrating by parts (cf. the identities (14) and (15)), we obtain (recalling that $u^{p} \in H_{0}^{1}(\Omega)$ )

$$
\begin{aligned}
\sum_{K \in T_{p h}} \int_{K} \nabla_{h} e^{p} \cdot \operatorname{curl} \varphi_{h} & =\int_{\Omega} \nabla u^{p} \cdot \operatorname{curl} \varphi_{h}-\sum_{K \in T_{p h}} \int_{K} \nabla_{h} u_{h}^{p} \cdot \operatorname{curl} \varphi_{h} \\
& =\int_{\Gamma} u^{p} \operatorname{curl} \varphi_{h} \cdot n-\sum_{K \in T_{p h}} \int_{\partial K} u_{h}^{p} \operatorname{curl} \varphi_{h} \cdot n_{K} \\
& =-\sum_{K \in T_{p h}} \int_{\partial K} u_{h}^{p} \operatorname{curl} \varphi_{h} \cdot n_{K} \\
& =-\sum_{E \in \mathcal{E}_{p h}} \int_{E} \llbracket u_{h}^{p} \rrbracket_{E} \operatorname{curl} \varphi_{h} \cdot n_{E} \\
& =-\sum_{E \in \mathcal{E}_{p h}}\left(\operatorname{curl} \varphi_{h} \cdot n_{E}\right) \int_{E} \llbracket u_{h}^{p} \rrbracket_{E},
\end{aligned}
$$

since the function $\left.\left(\operatorname{curl} \varphi_{h} \cdot n_{E}\right)\right|_{E}$ is constant on $E \in \mathcal{E}_{p h}$. The Crouzeix-Raviart property (6) satisfied by $u_{h}^{p} \in X_{p h}^{0}$ allows us to finish the proof. 
Lemma 3.4. The error $e^{p}$ satisfies

$$
\sum_{K \in T_{p h}} \int_{K} \nabla_{h} e^{p} \cdot \nabla w=\sum_{K \in T_{p h}} \int_{K}\left(f^{p}-\frac{u^{p}-u^{p-1}}{\tau_{p}}\right) w+\sum_{E \in \mathcal{E}_{p h}} \int_{E} J_{E, n}^{p} w
$$

for any $w \in H_{0}^{1}(\Omega)$.

Proof. Elementwise integration by parts and the observation that $\Delta u_{h}^{p}=0$ on all $K \in T_{p h}$ yield

$$
\begin{aligned}
\sum_{K \in T_{p h}} \int_{K} \nabla_{h} e^{p} \nabla w= & \int_{\Omega} \nabla u^{p} \cdot \nabla w-\sum_{K \in T_{p h}} \int_{K} \nabla_{h} u_{h}^{p} \cdot \nabla w \\
= & \int_{\Omega}\left(f^{p}-\frac{u^{p}-u^{p-1}}{\tau_{p}}\right) w \\
& -\sum_{K \in T_{p h}}\left(-\int_{K} \Delta u_{h}^{p} w+\int_{\partial K} n \cdot \nabla u_{h}^{p} w\right) \\
= & \int_{\Omega}\left(f^{p}-\frac{u^{p}-u^{p-1}}{\tau_{p}}\right) w \\
& -\sum_{K \in T_{p h}} \sum_{E \in \mathcal{E}_{K}} \int_{E} n \cdot \nabla u_{h}^{p} w .
\end{aligned}
$$

We conclude by using the definition of $J_{E, n}^{p}$ and the continuity of $w$ through the edges/faces.

Corollary 3.5. For any $w \in H_{0}^{1}(\Omega)$ and $\varphi \in H^{1}(\Omega)$ if $d=2$ and $\varphi \in H^{1}(\Omega)^{3}$ if $d=3$, we have

$$
\sum_{K \in T_{p h}} \int_{K} \nabla_{h} e^{p} \cdot(\nabla w+\operatorname{curl} \varphi)=\sum_{K \in T_{p h}} \int_{K}\left(f^{p}-\frac{u^{p}-u^{p-1}}{\tau_{p}}\right) w+\sum_{E \in \mathcal{E}_{p h}} \int_{E}\left(J_{E, n}^{p} w+J_{E, t}^{p} \cdot \varphi\right) .
$$

Proof. Direct consequence of Lemmas 3.2 and 3.4.

We now recall the following result (see Lem. 3.2 of [14] in 2D and [13] in 3D or [15], Chap. I):

Lemma 3.6 (Helmholtz decomposition of the error). We have the following error decomposition

$$
\nabla_{h} e^{p}=\nabla w^{p}+\operatorname{curl} \varphi^{p}
$$

with $\varphi^{p} \in H^{1}(\Omega)$ if $d=2$ and $\varphi^{p} \in\left(H^{1}(\Omega)\right)^{3}$ if $d=3$ and $w^{p} \in H_{0}^{1}(\Omega)$. Moreover the next estimates hold:

$$
\begin{aligned}
\left|w^{p}\right|_{1, \Omega} & \leq\left\|\nabla_{h} e^{p}\right\|, \\
\left|\varphi^{p}\right|_{1, \Omega} & \lesssim\left\|\nabla_{h} e^{p}\right\| .
\end{aligned}
$$

Proof. We consider the following Dirichlet problem: find $w^{p} \in H_{0}^{1}(\Omega)$ solution of

$$
\left\{\begin{array}{lr}
\boldsymbol{d i v}\left(\nabla_{h} e^{p}-\nabla w^{p}\right)=0 & \text { in } \Omega \\
w^{p}=0 & \text { on } \Gamma .
\end{array}\right.
$$

The weak formulation of that problem (23) is:

$$
\int_{\Omega} \nabla w^{p} \cdot \nabla v=\int_{\Omega} \nabla_{h} e^{p} \cdot \nabla v, \quad \forall v \in H_{0}^{1}(\Omega)
$$


As the vector field $\nabla_{h} e^{p}-\nabla w^{p}$ is divergence free in $\Omega$, i.e.,

$$
\boldsymbol{d i v}\left(\nabla_{h} e^{p}-\nabla w^{p}\right)=0 \text { in } \Omega .
$$

By Theorem I.3.1 of [15] if $d=2$ or Theorem I.3.4 of [15] if $d=3$, there exists $\varphi^{p} \in H^{1}(\Omega)$ if $d=2$ and $\varphi^{p} \in\left(H^{1}(\Omega)\right)^{3}$ if $d=3$ such that

$$
\operatorname{curl} \varphi^{p}=\nabla_{h} e^{p}-\nabla w^{p} .
$$

The estimate (21) directly follows by using (24) with $v=w^{p}$. The second estimate (22) is obtained as follows. Using the expansion (20), we may write

$$
\begin{aligned}
\int_{\Omega}\left|\operatorname{curl} \varphi^{p}\right|^{2} & =\int_{\Omega} \operatorname{curl} \varphi^{p} \cdot \operatorname{curl} \varphi^{p} \\
& =\int_{\Omega} \operatorname{curl} \varphi^{p} \cdot\left(\nabla_{h} e^{p}-\nabla w^{p}\right) .
\end{aligned}
$$

By Green's formula and the boundary conditions $w^{p}=0$ on $\Gamma$, we obtain

$$
\int_{\Omega}\left|\operatorname{curl} \varphi^{p}\right|^{2}=\int_{\Omega} \operatorname{curl} \varphi^{p} \cdot \nabla_{h} e^{p}
$$

By Cauchy-Schwarz's inequality we conclude

$$
\left\|\operatorname{curl} \varphi^{p}\right\| \leq\left\|\nabla_{h} e^{p}\right\| .
$$

If $d=2$ the estimate (22) directly follows from the above estimate since $\left|\varphi^{p}\right|_{1, \Omega}=\left\|\operatorname{curl} \varphi^{p}\right\|$. If $d=3$, we may notice that the application of the closed graph theorem yields a vector field $\varphi^{p}$ satisfying

$$
\left\|\varphi^{p}\right\|_{1, \Omega} \lesssim\left\|\operatorname{curl} \varphi^{p}\right\| .
$$

Indeed it suffices to consider the mapping

$$
F: H^{1}(\Omega)^{3} / K \rightarrow\left\{w \in L^{2}(\Omega)^{3}: \operatorname{div} w=0\right\}: \varphi \rightarrow \operatorname{curl} \varphi
$$

where $K=\left\{\varphi \in H^{1}(\Omega)^{3}:\right.$ curl $\left.\varphi=0\right\}$. This mapping is continuous and bijective (by Thm. I.3.4 of [15]) and consequently by the closed graph theorem, its inverse mapping is also continuous.

Therefore we may conclude as before using the above estimates (26) and (27).

The above lemmas allow us to prove the 
Lemma 3.7. The following identities hold

$$
\begin{aligned}
\tau_{p} \int_{\Omega} \nabla_{h} e^{p} \cdot \nabla w^{p}= & \tau_{p} \int_{\Omega}\left(f^{p}-\frac{u_{h}^{p}-u_{h}^{p-1}}{\tau_{p}}\right)\left(w^{p}-I_{C}^{0} w^{p}\right)+\tau_{p} \sum_{E \in \mathcal{E}_{p h}} \int_{E} J_{E, n}^{p}\left(w^{p}-I_{C}^{0} w^{p}\right)-\int_{\Omega}\left(e^{p}-e^{p-1}\right) w^{p} \\
\int_{\Omega} \nabla_{h} e^{p} \cdot \operatorname{curl} \varphi^{p}= & \sum_{E \in \mathcal{E}_{p h}} \int_{E} J_{E, t}^{p} \cdot\left(\varphi^{p}-I_{C} \varphi^{p}\right) \\
\left\|e^{p}\right\|^{2}+\tau_{p} \int_{\Omega}\left|\nabla_{h} e^{p}\right|^{2}= & \left(e^{p-1}, e^{p}\right)+\left(e^{p}-e^{p-1}, e^{p}-w^{p}-I_{C}^{0}\left(e^{p}-w^{p}\right)\right) \\
& +\tau_{p} \int_{\Omega} \nabla_{h} e^{p} \cdot \nabla I_{C}^{0}\left(e^{p}-w^{p}\right)+\tau_{p} \int_{\Omega}\left(f^{p}-\frac{u_{h}^{p}-u_{h}^{p-1}}{\tau_{p}}\right)\left(w^{p}-I_{C}^{0} w^{p}\right) \\
& +\tau_{p} \sum_{E \in \mathcal{E}_{p h}} \int_{E}\left(J_{E, n}^{p}\left(w^{p}-I_{C}^{0} w^{p}\right)+J_{E, t}^{p} \cdot\left(\varphi^{p}-I_{C} \varphi^{p}\right)\right)
\end{aligned}
$$

Proof. The identity (28) follows from the Galerkin relation of Lemma 3.1 with $v_{h}=I_{C}^{0} w^{p} \in V_{p h}^{0}$ and Lemma 3.4. The second identity (29) is a consequence of the orthogonality relation of Lemma 3.3 with $\varphi_{h}=I_{C} \varphi^{p}$ and Lemma 3.2.

Using the error decomposition (20) we may write

$$
\tau_{p} \int_{\Omega}\left|\nabla_{h} e^{p}\right|^{2}=\tau_{p} \int_{\Omega} \nabla_{h} e^{p} \cdot\left(\nabla w^{p}+\operatorname{curl} \varphi^{p}\right) .
$$

Therefore the identities (28), (29) directly lead to

$$
\begin{aligned}
\tau_{p} \int_{\Omega}\left|\nabla_{h} e^{p}\right|^{2}= & -\int_{\Omega}\left(e^{p}-e^{p-1}\right) w^{p}+\tau_{p} \int_{\Omega}\left(f^{p}-\frac{u_{h}^{p}-u_{h}^{p-1}}{\tau_{p}}\right)\left(w^{p}-I_{C}^{0} w^{p}\right) \\
& +\tau_{p} \sum_{E \in \mathcal{E}_{p h}} \int_{E}\left(J_{E, n}^{p}\left(w^{p}-I_{C}^{0} w^{p}\right)+J_{E, t}^{p} \cdot\left(\varphi^{p}-I_{C} \varphi^{p}\right)\right) .
\end{aligned}
$$

This identity may be equivalently written

$$
\begin{aligned}
\left\|e^{p}\right\|^{2}+\tau_{p} \int_{\Omega}\left|\nabla_{h} e^{p}\right|^{2}= & \left(e^{p-1}, e^{p}\right)+\left(e^{p}-e^{p-1}, e^{p}-w^{p}-I_{C}^{0}\left(e^{p}-w^{p}\right)\right)+\left(e^{p}-e^{p-1}, I_{C}^{0}\left(e^{p}-w^{p}\right)\right) \\
& +\tau_{p} \int_{\Omega}\left(f^{p}-\frac{u_{h}^{p}-u_{h}^{p-1}}{\tau_{p}}\right)\left(w^{p}-I_{C}^{0} w^{p}\right) \\
& +\tau_{p} \sum_{E \in \mathcal{E}_{p h}} \int_{E}\left(J_{E, n}^{p}\left(w^{p}-I_{C}^{0} w^{p}\right)+J_{E, t}^{p} \cdot\left(\varphi^{p}-I_{C} \varphi^{p}\right)\right) .
\end{aligned}
$$

This identity and the Galerkin orthogonality relation (16) lead to (30).

\section{A posteriori Analysis of the time Discretization}

Inspired from $[7,9,16,17]$, we define the time error indicators:

$$
\eta_{t}^{p}=\tau_{p}^{1 / 2}\left\|\nabla_{h}\left(u_{h}^{p}-u_{h}^{p-1}\right)\right\|, 1 \leq p \leq N .
$$


The only difference with the above papers relies on the fact that $u_{h}^{p}-u_{h}^{p-1}$ is not in $H^{1}(\Omega)$. Since the continuous problems (2) and (4) are not related to the approximation spaces $X_{p h}^{0}$, we easily adapt the arguments used there to our setting.

Note that in (31) we have written $\nabla_{h}\left(u_{h}^{p}-u_{h}^{p-1}\right)$ while $u_{h}^{p}$ and $u_{h}^{p-1}$ are not related to the same triangulation, but $u_{h}^{p}-u_{h}^{p-1}$ may be seen as a piecewise $\mathbb{P}_{1}$-function on the "triangulation" $T_{p h} \cap T_{p-1, h}$ made of the intersections of elements from $T_{p h}$ with elements from $T_{p-1, h}$. The broken gradient is then calculated on this triangulation $T_{p h} \cap T_{p-1, h}$.

For shortness we introduce the following notation: Denote by $\pi_{\tau} f$ the step function which is constant and equal to $f\left(t_{p}\right)$ on each interval $\left(t_{p-1}, t_{p}\right), 1 \leq p \leq N$. For a sequence $v^{p} \in X_{p h}^{0} \oplus H_{0}^{1}(\Omega), 0 \leq p \leq N$, we denote by $v_{\tau}$ its "Lagrange" interpolant, which is affine on each interval $\left[t_{p-1}, t_{p}\right], 1 \leq p \leq N$, and equal to $v^{p}$ at $t_{p}$, i.e., defined by,

$$
v_{\tau}(t)=\frac{t_{p}-t}{\tau_{p}} v^{p-1}+\frac{t-t_{p-1}}{\tau_{p}} v^{p}, \forall t \in\left[t_{p-1}, t_{p}\right], 1 \leq p \leq N
$$

Denote finally $e_{\tau}=u-u_{\tau}$, the time discretization error.

As

the semi-discrete equation (4) is equivalent to

$$
\partial_{t} u_{\tau}=\frac{u^{p}-u^{p-1}}{\tau_{p}} \text { on }\left(t_{p-1}, t_{p}\right)
$$

$$
\left(\partial_{t} u_{\tau}(t), v\right)+\left(\nabla u^{p}, \nabla v\right)=\left(f^{p}, v\right), \forall v \in H_{0}^{1}(\Omega), \forall t \in\left(t_{p-1}, t_{p}\right) .
$$

Taking the difference with (2), we derive the residual equation

$$
\left(\partial_{t} e_{\tau}(t), v\right)+\left(\nabla e_{\tau}(t), \nabla v\right)=\left(\left(f-f^{p}\right)(t), v\right)+\left(\nabla\left(u^{p}-u_{\tau}\right)(t), \nabla v\right), \forall v \in H_{0}^{1}(\Omega) \text {, } \forall \text { a.e. } t \in\left(t_{p-1}, t_{p}\right) .
$$

This equation allows to prove the

Theorem 4.1 (time upper error bound). The next estimate holds

$$
\left\|e_{\tau}\left(t_{n}\right)\right\|^{2}+\int_{0}^{t_{n}}\left\|\nabla e_{\tau}(s)\right\|^{2} \mathrm{~d} s \lesssim \sum_{p=1}^{n}\left(\eta_{t}^{p}\right)^{2}+\int_{0}^{t_{n}}\left\|\nabla_{h}\left(u_{\tau}-u_{h \tau}\right)(s)\right\|^{2} \mathrm{~d} s+\left\|f-\pi_{\tau} f\right\|_{L^{2}\left(0, t_{n} ; H^{-1}(\Omega)\right)}^{2} .
$$

Proof. The residual equation (33) yields (see Prop. 3.1 of [9])

$$
\left\|e_{\tau}\left(t_{n}\right)\right\|^{2}+\int_{0}^{t_{n}}\left\|\nabla e_{\tau}(t)\right\|^{2} \mathrm{~d} t \leq 2 \sum_{p=1}^{n} \int_{t_{p-1}}^{t_{p}}\left\|\nabla\left(u^{p}-u_{\tau}\right)(s)\right\|^{2} \mathrm{~d} s+2\left\|f-\pi_{\tau} f\right\|_{L^{2}\left(0, t_{n} ; H^{-1}(\Omega)\right)}^{2} .
$$

By the definition of $u_{\tau}$ we clearly have

$$
\int_{t_{p-1}}^{t_{p}}\left\|\nabla\left(u^{p}-u_{\tau}\right)(s)\right\|^{2} \mathrm{~d} s=\frac{\tau_{p}}{3}\left\|\nabla\left(u^{p}-u^{p-1}\right)\right\|^{2} .
$$

Using the triangular inequality, we simply write

$$
\tau_{p}^{1 / 2}\left\|\nabla\left(u^{p}-u^{p-1}\right)\right\| \leq \eta_{t}^{p}+\tau_{p}^{1 / 2}\left\|\nabla_{h}\left(u^{p}-u_{h}^{p}\right)\right\|+\tau_{p}^{1 / 2}\left\|\nabla_{h}\left(u_{h}^{p-1}-u_{h}^{p-1}\right)\right\| .
$$

Moreover the arguments from Lemma 2.3 of [9] yields

$$
\tau_{p}\left\|\nabla_{h}\left(u^{p}-u_{h}^{p}\right)\right\|^{2}+\tau_{p}\left\|\nabla_{h}\left(u_{h}^{p-1}-u_{h}^{p-1}\right)\right\|^{2} \lesssim \int_{t_{p-1}}^{t_{p}}\left\|\nabla_{h}\left(u_{\tau}-u_{h \tau}\right)(s)\right\|^{2} \mathrm{~d} s .
$$


The above identity and these two estimates yield

$$
\int_{t_{p-1}}^{t_{p}}\left\|\nabla\left(u^{p}-u_{\tau}\right)(s)\right\|^{2} \mathrm{~d} s \lesssim\left(\eta_{t}^{p}\right)^{2}+\int_{t_{p-1}}^{t_{p}}\left\|\nabla_{h}\left(u_{\tau}-u_{h \tau}\right)(s)\right\|^{2} \mathrm{~d} s
$$

This estimate in (35) leads to the conclusion.

Corollary 4.2 (second time upper error bound). The next estimate holds

$$
\left\|\partial_{t} e_{\tau}\right\|_{L^{2}\left(0, t_{n} ; H^{-1}(\Omega)\right)}^{2} \lesssim \sum_{p=1}^{n}\left(\eta_{t}^{p}\right)^{2}+\int_{0}^{t_{n}}\left\|\nabla_{h}\left(u_{\tau}-u_{h \tau}\right)(s)\right\|^{2} \mathrm{~d} s+\left\|f-\pi_{\tau} f\right\|_{L^{2}\left(0, t_{n} ; H^{-1}(\Omega)\right)}^{2}
$$

Proof. The residual equation (33) directly gives

$$
\left\|\partial_{t} e_{\tau}\right\|_{L^{2}\left(0, t_{n} ; H^{-1}(\Omega)\right)}^{2} \lesssim\left\|f-\pi_{\tau} f\right\|_{L^{2}\left(0, t_{n} ; H^{-1}(\Omega)\right)}^{2}+\int_{0}^{t_{n}}\left\|\nabla e_{\tau}(s)\right\|^{2} \mathrm{~d} s+\sum_{p=1}^{n} \int_{t_{p-1}}^{t_{p}}\left\|\nabla\left(u^{p}-u_{\tau}\right)(s)\right\|^{2} \mathrm{~d} s .
$$

The second term of this right-hand side is estimated in (34), while the third term is estimated via (38).

The local time upper bound is even easier to prove:

Theorem 4.3 (time lower error bound). For all $p=1, \cdots, N$, the next estimate holds

$$
\begin{aligned}
\eta_{t}^{p} \lesssim\left\|\nabla_{h} e_{\tau}\right\|_{L^{2}\left(t_{p-1}, t_{p} ; L^{2}(\Omega)\right)} & +\left\|\partial_{t} e_{\tau}\right\|_{L^{2}\left(t_{p-1}, t_{p} ; H^{-1}(\Omega)\right)} \\
& +\tau_{p}^{1 / 2}\left(\left\|\nabla_{h}\left(u^{p}-u_{h}^{p}\right)\right\|+\left\|\nabla_{h}\left(u^{p-1}-u_{h}^{p-1}\right)\right\|\right)+\left\|f-\pi_{\tau} f\right\|_{L^{2}\left(t_{p-1}, t_{p} ; H^{-1}(\Omega)\right)} .
\end{aligned}
$$

Proof. By the triangular inequality we may write

$$
\eta_{t}^{p} \lesssim \tau_{p}^{1 / 2}\left(\left\|\nabla\left(u^{p}-u^{p-1}\right)\right\|+\left\|\nabla_{h}\left(u^{p}-u_{h}^{p}\right)\right\|+\left\|\nabla_{h}\left(u^{p-1}-u_{h}^{p-1}\right)\right\|\right) .
$$

The estimation of the term $\tau_{p}^{1 / 2}\left\|\nabla\left(u^{p}-u^{p-1}\right)\right\|$ is made as in Proposition 3.3 of [9] by using the identity (36) (with $n=p$ ) and taking $v=u^{p}-u_{\tau}$ in (33) and integrating the result in $t \in\left(t_{p-1}, t_{p}\right)$.

\section{A POSTERIORI ANALYsis OF the SPATial Discretization}

\subsection{Upper error bound}

The exact element residual is given by

$$
f^{p}-\frac{u_{h}^{p}-u_{h}^{p-1}}{\tau_{p}}
$$

As usual [20] it is replaced by an approximate element residual

$$
f_{h}^{p}-\frac{u_{h}^{p}-u_{h}^{p-1}}{\tau_{p}}
$$

where $f_{h}^{p}$ is a finite dimensional approximation of $f^{p}$ (for instance $\left(f_{h}^{p}\right)_{\mid K}:=\frac{1}{|K|} \int_{K} f^{p}$, for all $K \in T_{p h}$ ).

Definition 5.1. Let $p \geq 1$. The local error estimator $\eta_{K}^{p}$ is defined by

$$
\eta_{K}^{p}=h_{K}\left\|f_{h}^{p}-\frac{u_{h}^{p}-u_{h}^{p-1}}{\tau_{p}}\right\|_{K}+\sum_{E \in \mathcal{E}_{K}} h_{E}^{1 / 2}\left(\left\|J_{E, n}^{p}\right\|_{E}+\left\|J_{E, t}^{p}\right\|_{E}\right) .
$$


The global spatial error estimator $\eta^{p}$ is given by

$$
\left(\eta^{p}\right)^{2}=\sum_{K \in T_{p h}}\left(\eta_{K}^{p}\right)^{2}
$$

The local and global approximation terms are defined by

$$
\xi_{K}^{p}=h_{K}\left\|f^{p}-f_{h}^{p}\right\|_{\omega_{K}}, \quad\left(\xi^{p}\right)^{2}=\sum_{K \in T_{p h}}\left(\xi_{K}^{p}\right)^{2} .
$$

Theorem 5.2 (upper error bound). The next estimate holds

$$
\left\|e^{n}\right\|^{2}+\sum_{p=1}^{n} \tau_{p}\left\|\nabla_{h} e^{p}\right\|^{2} \lesssim \sum_{p=1}^{n} \max \left\{h_{p}^{2}, \tau_{p}\right\}\left(\eta^{p}\right)^{2}+\left\|e^{0}\right\|^{2}+\sum_{p=1}^{n} \tau_{p}\left(\xi^{p}\right)^{2} .
$$

Proof. This upper bound is a consequence of Lemmas 3.6 and 3.7. We first estimate some terms of the righthand side of the identity (30) of Lemma 3.7. Using successively Cauchy-Schwarz's inequality, the estimate (11) and the definition 5.1 of the local estimator, we obtain

$$
\begin{aligned}
\sum_{K \in T_{p h}} \int_{K}\left(f_{h}^{p}-\frac{u_{h}^{p}-u_{h}^{p-1}}{\tau_{p}}\right)\left(w^{p}-I_{C}^{0} w^{p}\right) & \lesssim \sum_{K \in T_{p h}} h_{K}\left\|f_{h}^{p}-\frac{u_{h}^{p}-u_{h}^{p-1}}{\tau_{p}}\right\|_{K}\left|w^{p}\right|_{1, \tilde{\omega}_{K}} \\
& \lesssim \sum_{K \in T_{p h}} \eta_{K}^{p}\left|w^{p}\right|_{1, \tilde{\omega}_{K}} .
\end{aligned}
$$

By discrete Cauchy-Schwarz's inequality we get

$$
\sum_{K \in T_{p h}} \int_{K}\left(f_{h}^{p}-\frac{u_{h}^{p}-u_{h}^{p-1}}{\tau_{p}}\right)\left(w^{p}-I_{C}^{0} w^{p}\right) \lesssim \eta^{p}\left|w^{p}\right|_{1, \Omega} .
$$

Similarly using (12) and (10) we estimate the term with the jumps of normal and tangential derivatives:

$$
\begin{aligned}
\sum_{E \in \mathcal{E}_{p h}} \int_{E} J_{E, n}^{p}\left(w^{p}-I_{C}^{0} w^{p}\right) & \leq \sum_{E \in \mathcal{E}_{p h}}\left\|J_{E, n}^{p}\right\|_{E}\left\|w^{p}-I_{C}^{0} w^{p}\right\|_{E} \\
& \lesssim \sum_{E \in \mathcal{E}_{p h}}\left\|J_{E, n}^{p}\right\|_{E} h_{E}^{1 / 2}\left|w^{p}\right|_{1, \tilde{\omega}_{E}} \\
& \lesssim \sum_{K \in T_{p h}} \eta_{K}^{p}\left|w^{p}\right|_{1, \tilde{\omega}_{K}}, \\
\sum_{E \in \mathcal{E}_{p h}} \int_{E} J_{E, t}^{p} \cdot\left(\varphi^{p}-I_{C} \varphi^{p}\right) & \leq \sum_{E \in \mathcal{E}_{p h}}\left\|J_{E, t}^{p}\right\|_{E}\left\|\varphi^{p}-I_{C} \varphi^{p}\right\|_{E} \\
& \lesssim \sum_{E \in \mathcal{E}_{p h}}\left\|J_{E, t}^{p}\right\|_{E} h_{E}^{1 / 2}\left|\varphi^{p}\right|_{1, \tilde{\omega}_{E}} \\
& \lesssim \sum_{K \in T_{p h}} \eta_{K}^{p}\left|\varphi^{p}\right|_{1, \tilde{\omega}_{K}} .
\end{aligned}
$$


As before discrete Cauchy-Schwarz's inequality yields

$$
\begin{gathered}
\sum_{E \in \mathcal{E}_{p h}} \int_{E} J_{E, n}^{p}\left(w^{p}-I_{C}^{0} w^{p}\right) \lesssim \eta^{p}\left|w^{p}\right|_{1, \Omega}, \\
\sum_{E \in \mathcal{E}_{p h}} \int_{E} J_{E, t}^{p} \cdot\left(\varphi^{p}-I_{C} \varphi^{p}\right) \lesssim \eta^{p}\left|\varphi^{p}\right|_{1, \Omega} .
\end{gathered}
$$

Again (11) allows to estimate the term:

$$
\sum_{K \in T_{p h}} \int_{K}\left(f^{p}-f_{h}^{p}\right)\left(w^{p}-I_{C}^{0} w^{p}\right) \leq \sum_{K \in T_{p h}} h_{K}\left\|f^{p}-f_{h}^{p}\right\|_{K}\left|w^{p}\right|_{1, \tilde{\omega}_{K}}
$$

and consequently

$$
\sum_{K \in T_{p h}} \int_{K}\left(f^{p}-f_{h}^{p}\right)\left(w^{p}-I_{C}^{0} w^{p}\right) \lesssim \xi^{p}\left|w^{p}\right|_{1, \Omega}
$$

Applying Cauchy-Schwarz's inequality and the estimate (11) we get

$$
\left|\left(e^{p}-e^{p-1}, e^{p}-w^{p}-I_{C}^{0}\left(e^{p}-w^{p}\right)\right)\right| \lesssim h_{p}\left\|e^{p}-e^{p-1}\right\|\left\|\nabla_{h}\left(e^{p}-w^{p}\right)\right\| .
$$

The above estimates and (13) in the identity (30) yield

$$
\begin{aligned}
\left\|e^{p}\right\|^{2}+\tau_{p} \int_{\Omega}\left|\nabla_{h} e^{p}\right|^{2} \leq & \left(e^{p-1}, e^{p}\right)+C h_{p}\left\|e^{p}-e^{p-1}\right\|\left\|\nabla_{h}\left(e^{p}-w^{p}\right)\right\| \\
& +C \tau_{p}\left\|\nabla_{h} e^{p}\right\|\left\|\nabla_{h}\left(e^{p}-w^{p}\right)\right\|+C \tau_{p} \eta^{p}\left|\varphi^{p}\right|_{1, \Omega}+C \tau_{p}\left(\eta^{p}+\xi^{p}\right)\left|w^{p}\right|_{1, \Omega}
\end{aligned}
$$

for some constant $C>0$ depending only on the minimal angle of $T_{p h}$.

This estimate does not directly yield the desired estimate due to the factors $\left\|\nabla_{h}\left(e^{p}-w^{p}\right)\right\|,\left|w^{p}\right|_{1, \Omega}$ and $\left|\varphi^{p}\right|_{1, \Omega}$. We therefore need to estimate these factors. We first start with this last one. Using the identities (25) and (29) we may write

$$
\int_{\Omega}\left|\operatorname{curl} \varphi^{p}\right|^{2}=\sum_{E \in \mathcal{E}_{p h}} \int_{E} J_{E, t}^{p} \cdot\left(\varphi^{p}-I_{C} \varphi^{p}\right) .
$$

Using the approximation error estimate (10) and the definition of the a posteriori error estimator we get

$$
\left\|\operatorname{curl} \varphi^{p}\right\|^{2} \lesssim \eta^{p}\left|\varphi^{p}\right|_{1, \Omega}
$$

With the help of (27) if $d=3$, we conclude that

$$
\left|\varphi^{p}\right|_{1, \Omega} \lesssim \eta^{p}
$$

For the estimation of the norm of $\nabla_{h}\left(e^{p}-w^{p}\right)$, we start with

$$
\left\|\nabla_{h}\left(e^{p}-w^{p}\right)\right\|^{2}=\int_{\Omega} \nabla_{h}\left(e^{p}-w^{p}\right) \cdot \nabla_{h}\left(e^{p}-w^{p}\right) .
$$

Using the Helmholtz decomposition (20) we then write

$$
\left\|\nabla_{h}\left(e^{p}-w^{p}\right)\right\|^{2}=\int_{\Omega} \nabla_{h}\left(e^{p}-w^{p}\right) \cdot \operatorname{curl} \varphi^{p} .
$$


By Lemma 3.7 and Green's formula (recalling that $w^{p}=0$ on $\Gamma$ ), we arrive at

$$
\left\|\nabla_{h}\left(e^{p}-w^{p}\right)\right\|^{2}=\sum_{E \in \mathcal{E}_{p h}} \int_{E} J_{E, t}^{p} \cdot\left(\varphi^{p}-I_{C} \varphi^{p}\right)
$$

Using the estimates (44) and (47) we obtain

$$
\left\|\nabla_{h}\left(e^{p}-w^{p}\right)\right\| \lesssim \eta^{p}
$$

By the triangular inequality we have

$$
\left\|\nabla w^{p}\right\| \leq\left\|\nabla_{h}\left(w^{p}-e^{p}\right)\right\|+\left\|\nabla_{h} e^{p}\right\|,
$$

and by the well-known estimate $(a+b)^{2} \leq 2 a^{2}+2 b^{2}$, valid for any real numbers $a, b$, we obtain

$$
\left\|\nabla w^{p}\right\|^{2} \leq 2\left\|\nabla_{h}\left(w^{p}-e^{p}\right)\right\|^{2}+2\left\|\nabla_{h} e^{p}\right\|^{2} .
$$

By the estimate (48) we arrive at

$$
\left\|\nabla w^{p}\right\|^{2} \leq C\left(\eta^{p}\right)^{2}+2\left\|\nabla_{h} e^{p}\right\|^{2}
$$

for some constant $C>0$ depending only on the minimal angle of $T_{p h}$.

We are now able to conclude: Using the estimates (47), (48) and Young's inequality in (46), we may write

$$
\left.\int_{\Omega}\left|e^{p}\right|^{2}+\tau_{p} \int_{\Omega}\left|\nabla_{h} e^{p}\right|^{2} \leq\left(e^{p-1}, e^{p}\right)+C h_{p}\left\|e^{p}-e^{p-1}\right\| \eta^{p}+C \tau_{p}\left\|\nabla_{h} e^{p}\right\| \eta^{p}+C \tau_{p}\left(\left(\eta^{p}\right)^{2}+\xi^{p}\right)^{2}\right)+\frac{1}{8} \tau_{p}\left|w^{p}\right|_{1, \Omega}^{2},
$$

for some constant $C>0$ depending only on the minimal angle of $T_{p h}$.

Using the estimate (49) for the estimate of the term $\left|w^{p}\right|_{1, \Omega}^{2}$ and again Young's inequality, we finally arrive at

$$
\begin{aligned}
\int_{\Omega}\left|e^{p}\right|^{2}+\tau_{p} \int_{\Omega}\left|\nabla_{h} e^{p}\right|^{2} & \left.\leq\left(e^{p-1}, e^{p}\right)+\frac{1}{2}\left\|e^{p}-e^{p-1}\right\|^{2}+C h_{p}^{2}\left(\eta^{p}\right)^{2}+C \tau_{p}\left(\left(\eta^{p}\right)^{2}+\xi^{p}\right)^{2}\right)+\frac{\tau_{p}}{2}\left\|\nabla_{h} e^{p}\right\|^{2} \\
& \leq \frac{1}{2}\left\|e^{p}\right\|^{2}+\frac{1}{2}\left\|e^{p-1}\right\|^{2}+C\left(\max \left\{h_{p}^{2}, \tau_{p}\right\}\left(\eta^{p}\right)^{2}+\tau_{p}\left(\xi^{p}\right)^{2}\right)+\frac{\tau_{p}}{2}\left\|\nabla_{h} e^{p}\right\|^{2}
\end{aligned}
$$

for some constant $C>0$ depending only on the minimal angle of $T_{p h}$. This estimate is equivalent to

$$
\left\|e^{p}\right\|^{2}+\tau_{p} \int_{\Omega}\left|\nabla_{h} e^{p}\right|^{2} \leq\left\|e^{p-1}\right\|^{2}+2 C\left(\max \left\{h_{p}^{2}, \tau_{p}\right\}\left(\eta^{p}\right)^{2}+\tau_{p}\left(\xi^{p}\right)^{2}\right)
$$

and we conclude by taking the sum on $p=1, \ldots, n$.

Corollary 5.3 (second upper error bound). The next estimate holds

$$
\left\|\partial_{t}\left(u_{\tau}-u_{h \tau}\right)\right\|_{L^{2}\left(0, t_{n} ; H^{-1}(\Omega)\right.}^{2} \lesssim \sum_{p=1}^{n} \max \left\{h_{p}^{2}, \tau_{p}\right\}\left(\eta^{p}\right)^{2}+\left\|e^{0}\right\|^{2}+\left\|\nabla_{h} e^{0}\right\|^{2}+\sum_{p=1}^{n} \tau_{p}\left(\xi^{p}\right)^{2} .
$$

Proof. By definition we have

$$
\left\|\partial_{t}\left(u_{\tau}-u_{h \tau}\right)(t)\right\|_{H^{-1}(\Omega)}=\sup _{v \in H_{0}^{1}(\Omega)} \frac{\left(\partial_{t}\left(u_{\tau}-u_{h \tau}\right)(t), v\right)}{\|v\|_{1, \Omega}}
$$


Using the property

$$
\partial_{t}\left(u_{\tau}-u_{h \tau}\right)(t)=\frac{e^{p}-e^{p-1}}{\tau_{p}}, \forall t \in\left(t_{p-1}, t_{p}\right)
$$

and the semi-discrete equation (4), for any $t \in\left(t_{p-1}, t_{p}\right)$ we may write

$$
\left(\partial_{t}\left(u_{\tau}-u_{h \tau}\right)(t), v\right)=R^{p}(v)-\left(\nabla_{h} e^{p}, \nabla v\right),
$$

where the residual $R^{p}$ is defined by

$$
R^{p}(v)=\left(f^{p}, v\right)-\left(\frac{u_{h}^{p}-u_{h}^{p-1}}{\tau_{p}}, v\right)-\left(\nabla_{h} u_{h}^{p}, \nabla v\right), \forall v \in H_{0}^{1}(\Omega) .
$$

As (5) implies that

the above identity becomes

$$
R^{p}\left(v_{h}\right)=0, \forall v_{h} \in V_{n h}^{0}
$$

$$
\left(\partial_{t}\left(u_{\tau}-u_{h \tau}\right)(t), v\right)=R^{p}\left(v-v_{h}\right)-\left(\nabla_{h} e^{p}, \nabla v\right), \forall v_{h} \in V_{p h}^{0}, t \in\left(t_{p-1}, t_{p}\right) .
$$

Taking $v_{h}=I_{C} v$, applying Green's formula componentwise, and using the estimate (12) we get

$$
\left|\left(\partial_{t}\left(u_{\tau}-u_{h \tau}\right)(t), v\right)\right| \lesssim\left(\eta^{p}+\left\|\nabla_{h} e^{p}\right\|\right)\|\nabla v\|, \forall t \in\left(t_{p-1}, t_{p}\right) .
$$

This estimate and Poincaré-Friedrichs' inequality lead to

$$
\left\|\partial_{t}\left(u_{\tau}-u_{h \tau}\right)(t)\right\|_{H^{-1}(\Omega)} \lesssim \eta^{p}+\left\|\nabla_{h} e^{p}\right\|, \forall t \in\left(t_{p-1}, t_{p}\right) .
$$

The conclusion then follows from the estimate (41).

\subsection{Lower error bound}

We establish the lower error bound of the estimator $\eta_{K}^{p}$ in a more or less standard way (see $[14,20]$ ). Since we consider a nonstationary problem, we further need the following assumption (see $[9,22])$, that is easily checked in an adaptive context:

Assumption 5.4. For all $1 \leq p \leq N$, there exists a conforming triangulation $\tilde{T}_{p h}$ such that each element $K$ of $T_{p-1, h}$ or of $T_{p h}$ is the union of elements $\tilde{K}$ of $\tilde{T}_{p h}$ such that $h_{K} \sim h_{\tilde{K}}$.

For our convenience we reformulate Corollary 3.5 in the following way:

Lemma 5.5. For all $v \in H_{0}^{1}(\Omega), \varphi \in H^{1}(\Omega)$ if $d=2$ and $\varphi \in H^{1}(\Omega)^{3}$ if $d=3$ we have the following identity:

$$
\begin{aligned}
\int_{\Omega}\left(e^{p}-e^{p-1}\right) v+\tau_{p} \sum_{K \in T_{p h}} \int_{K} \nabla_{h} e^{p} \cdot(\nabla v+\operatorname{curl} \varphi) & =\tau_{p} \int_{\Omega}\left(f^{p}-f_{h}^{p}\right) v \\
& +\tau_{p} \sum_{K \in T_{p h}} \int_{K}\left(f_{h}^{p}-\frac{u_{h}^{p}-u_{h}^{p-1}}{\tau_{p}}\right) v+\tau_{p} \sum_{E \in \mathcal{E}_{p h}} \int_{E}\left(J_{E, n}^{p} v+J_{E, t}^{p} \cdot \varphi\right) .
\end{aligned}
$$

Theorem 5.6 (local lower error bound). If Assumption 5.4 holds, then for all $1 \leq p \leq N$, it holds

$$
\eta_{K}^{p} \lesssim h_{K}\left\|\frac{e^{p}-e^{p-1}}{\tau_{p}}\right\|_{\omega_{K}}+\left\|\nabla_{h} e^{p}\right\|_{\omega_{K}}+\xi_{K}^{p} .
$$


Proof. Element residual Fix an arbitrary element $K \in \tilde{T}_{p h}$ and define

$$
r_{K}^{p}:=\left(f_{h}^{p}-\frac{u_{h}^{p}-u_{h}^{p-1}}{\tau_{p}}\right)_{\mid K}, \quad w_{K}^{p}:=b_{K} r_{K}^{p},
$$

where $b_{K}=\lambda_{1}^{K} \lambda_{2}^{K} \lambda_{3}^{K}$ is the standard bubble function associated with $K$ (see e.g. [20]). Standard inverse inequalities (cf. [20]) and Lemma 5.5 with $v=w_{K}^{p}$ and $\varphi=0$ give

$$
\begin{aligned}
\left\|r_{K}^{p}\right\|_{K}^{2} \sim \int_{K} r_{K}^{p} w_{K}^{p} & =\int_{K}\left(f_{h}^{p}-\frac{u_{h}^{p}-u_{h}^{p-1}}{\tau_{p}}\right) w_{K}^{p} \\
& =\int_{K}\left(\frac{e^{p}-e^{p-1}}{\tau_{p}} w_{K}^{p}+\nabla_{h} e^{p} \cdot \nabla w_{K}^{p}-\left(f^{p}-f_{h}^{p}\right) w_{K}^{p}\right) \\
& \lesssim\left(\left\|\frac{e^{p}-e^{p-1}}{\tau_{p}}\right\|_{K}+h_{K}^{-1}\left|e^{p}\right|_{1, K}+\left\|f^{p}-f_{h}^{p}\right\|_{K}\right)\left\|r_{K}^{p}\right\|_{K} .
\end{aligned}
$$

This proves the estimate

$$
h_{K}\left\|r_{K}^{p}\right\|_{K} \lesssim h_{K}\left\|\frac{e^{p}-e^{p-1}}{\tau_{p}}\right\|_{K}+\left|e^{p}\right|_{1, K}+h_{K}\left\|f^{p}-f_{h}^{p}\right\|_{K}, \forall K \in \tilde{T}_{p h} .
$$

Now for $K \in T_{p h}$, the Assumption 5.4 yields

$$
h_{K}^{2}\left\|r_{K}^{p}\right\|_{K}^{2} \lesssim \sum_{\tilde{K} \in \tilde{T}_{p h}: \tilde{K} \subset K} h_{\tilde{K}}^{2}\left\|r_{\tilde{K}}^{p}\right\|_{\tilde{K}}^{2} .
$$

Using the estimate (52) and the fact that $h_{\tilde{K}} \leq h_{K}$ for $\tilde{K} \subset K$ we have proved that

$$
h_{K}\left\|r_{K}^{p}\right\|_{K} \lesssim h_{K}\left\|\frac{e^{p}-e^{p-1}}{\tau_{p}}\right\|_{K}+\left|e^{p}\right|_{1, K}+\xi_{K}, \forall K \in T_{p h} .
$$

Tangential jump. Next we consider an arbitrary edge/face $E$ of $T_{p h}$ and define

$$
w_{E}^{p}:=b_{E} J_{E, t}^{p},
$$

where $b_{E}$ is the standard bubble function associated with $E$ (see e.g. [20]).

Lemma 5.5 with $v=0$ and $\varphi=w_{E}^{p}$ and inverse inequalities give

$$
\begin{aligned}
\left\|J_{E, t}^{p}\right\|_{E}^{2} & \sim \sum_{K \subset \omega_{E}} \int_{K} \nabla_{h} e^{p} \cdot \text { curl } w_{E}^{p} \\
& \lesssim\left\|\nabla_{h} e^{p}\right\| \omega_{E}\left\|\nabla w_{E}^{p}\right\|_{\omega_{E}} \\
& \lesssim h_{E}^{-1 / 2}\left\|J_{E, t}^{p}\right\|_{E}\left\|\nabla_{h} e^{p}\right\|_{\omega_{E}}
\end{aligned}
$$

This proves that

$$
h_{E}^{1 / 2}\left\|J_{E, t}^{p}\right\|_{E} \lesssim\left\|\nabla_{h} e^{p}\right\|_{\omega_{E}} .
$$

Normal jump. Similarly for an interior arbitrary edge/face $E$ of $T_{p h}$, we define

$$
w_{E}^{p}:=b_{E} J_{E, n}^{p} .
$$


Using inverse estimates and Lemma 5.5 with $v=w_{E}^{p}$ and $\varphi=0$ we obtain

$$
\left\|J_{E, n}^{p}\right\|_{E} \lesssim h_{E}^{1 / 2}\left\|\frac{e^{p}-e^{p-1}}{\tau_{p}}\right\|_{\omega_{E}}+h_{E}^{-1 / 2}\left\|\nabla_{h} e^{p}\right\|_{\omega_{E}}+h_{E}^{1 / 2}\left\|f^{p}-f_{h}^{p}\right\|_{\omega_{E}}+h_{E}^{1 / 2}\left\|f_{h}^{p}-\frac{u_{h}^{p}-u_{h}^{p-1}}{\tau_{p}}\right\|_{\omega_{E}} .
$$

With the help of (53) this yields

$$
h_{E}^{1 / 2}\left\|J_{E, n}^{p}\right\|_{E} \lesssim h_{E}\left\|\frac{e^{p}-e^{p-1}}{\tau_{p}}\right\|_{\omega_{E}}+\left\|\nabla_{h} e^{p}\right\|_{\omega_{E}}+h_{E}\left\|f^{p}-f_{h}^{p}\right\|_{\omega_{E}} .
$$

The conclusion follows from the estimates (53), (54) and (55).

Corollary 5.7 (second local lower error bound). If Assumption 5.4 holds, then for all $1 \leq p \leq N$, it holds

$$
\left(\eta^{p}\right)^{2} \lesssim\left\|\partial_{t}\left(u_{\tau}-u_{h \tau}\right)\right\|_{H^{-1}(\Omega)}^{2}+\left\|\nabla_{h} e^{p}\right\|^{2}+\left(\xi^{p}\right)^{2}
$$

Proof. As

$$
\partial_{t}\left(u_{\tau}-u_{h \tau}\right)(t)=\frac{e^{p}-e^{p-1}}{\tau_{p}}, \forall t \in\left(t_{p-1}, t_{p}\right)
$$

in the above proof we need to replace the local $L^{2}$-norm of $\frac{e^{p}-e^{p-1}}{\tau_{p}}$ by its global $H^{-1}(\Omega)$-norm. For that purpose in Lemma 5.5 we take $\varphi=0$ and

$$
v=\sum_{\tilde{K} \in \tilde{T}_{p h}} h_{\tilde{K}}^{2} r_{\tilde{K}}^{p} b_{\tilde{K}}
$$

which yields, with the help of the assumption 5.4,

$$
\sum_{K \in T_{p h}} h_{K}^{2}\left\|r_{K}^{p}\right\|_{K}^{2} \lesssim\left(\left\|\partial_{t}\left(u_{\tau}-u_{h \tau}\right)\right\|_{H^{-1}(\Omega)}+\left\|\nabla_{h} e^{p}\right\|\right)\|\nabla v\|+\sum_{K \in T_{p h}}\left\|f^{p}-f_{h}^{p}\right\|_{K}\|v\|_{K}
$$

Standard inverse inequalities lead to

$$
\sum_{K \in T_{p h}} h_{K}^{2}\left\|r_{K}^{p}\right\|_{K}^{2} \lesssim\left\|\partial_{t}\left(u_{\tau}-u_{h \tau}\right)\right\|_{H^{-1}(\Omega)}^{2}+\left\|\nabla_{h} e^{p}\right\|^{2}+\left(\xi^{p}\right)^{2} .
$$

Similarly for the estimate of the normal jump, we use Lemma 5.5 with $\varphi=0$ and

$$
v=\sum_{E \in \mathcal{E}_{p h}^{i n t}} h_{E} J_{E, n}^{p} b_{E}
$$

to get

$$
\sum_{E \in \mathcal{E}_{p h}^{i n t}} h_{E}\left\|J_{E, n}^{p}\right\|_{E}^{2} \lesssim\left\|\partial_{t}\left(u_{\tau}-u_{h \tau}\right)\right\|_{H^{-1}(\Omega)}^{2}+\left\|\nabla_{h} e^{p}\right\|^{2}+\left(\xi^{p}\right)^{2} .
$$

These estimates and (54) allow to conclude. 


\section{A posteriori AnAlysis of the Full Discretization}

For all $n=1, \cdots, N$, denote the full error $E\left(t_{n}\right)$ at time $t_{n}$ by

$$
\begin{aligned}
E\left(t_{n}\right)^{2}= & \left\|u\left(t_{n}\right)-u_{h}^{n}\right\|^{2}+\left\|u^{n}-u_{h}^{n}\right\|^{2}+\left\|\partial_{t}\left(u-u_{\tau}\right)\right\|_{L^{2}\left(0, t_{n} ; H^{-1}(\Omega)\right)}^{2}+\left\|\partial_{t}\left(u_{\tau}-u_{h \tau}\right)\right\|_{L^{2}\left(0, t_{n} ; H^{-1}(\Omega)\right)}^{2} \\
& +\int_{0}^{t_{n}}\left\|\nabla_{h}\left(u-u_{\tau}\right)(\cdot, s)\right\|^{2} \mathrm{~d} s+\int_{0}^{t_{n}}\left\|\nabla_{h}\left(u_{\tau}-u_{h \tau}\right)(\cdot, s)\right\|^{2} \mathrm{~d} s .
\end{aligned}
$$

Combining the results from the previous sections, we get the following global upper and lower bounds:

Theorem 6.1 (full error bounds). For any $n=1, \cdots, N$, the next upper error bound holds:

$$
E\left(t_{n}\right)^{2} \lesssim \sum_{p=1}^{n}\left(\left(\eta_{t}^{p}\right)^{2}+\max \left\{h_{p}^{2}, \tau_{p}\right\}\left(\eta^{p}\right)^{2}\right)+\left\|f-\pi_{\tau} f\right\|_{L^{2}\left(0, t_{n} ; H^{-1}(\Omega)\right)}^{2}+\sum_{p=1}^{n} \tau_{p}\left(\xi^{p}\right)^{2}+\left\|e^{0}\right\|^{2}+\tau_{0}\left\|\nabla_{h} e^{0}\right\|^{2} .
$$

If moreover Assumption 5.4 holds, then for any $n=1, \cdots, N$, the next lower error bound holds:

$$
\sum_{p=1}^{n}\left(\left(\eta_{t}^{p}\right)^{2}+\tau_{p}\left(\eta^{p}\right)^{2}\right) \lesssim E\left(t_{n}\right)^{2}+\left\|f-\pi_{\tau} f\right\|_{L^{2}\left(0, t_{n} ; H^{-1}(\Omega)\right)}^{2}+\sum_{p=1}^{n} \tau_{p}\left(\xi^{p}\right)^{2}
$$

Proof. Let us start with the upper error bound. First by Theorem 4.1 and Corollary 4.2, we have

$$
\begin{aligned}
E\left(t_{n}\right)^{2} \lesssim & \sum_{p=1}^{n}\left(\eta_{t}^{p}\right)^{2}+\left\|\left(u_{\tau}-u_{h \tau}\right)\left(t_{n}\right)\right\|^{2}+\int_{0}^{t_{n}} \| \nabla_{h}\left(u_{\tau}-u_{h \tau}(s) \|^{2} \mathrm{~d} s\right. \\
& +\left\|\partial_{t}\left(u_{\tau}-u_{h \tau}\right)\right\|_{L^{2}\left(0, t_{n} ; H^{-1}(\Omega)\right)}^{2}+\left\|f-\pi_{\tau} f\right\|_{L^{2}\left(0, t_{n} ; H^{-1}(\Omega)\right)}^{2}
\end{aligned}
$$

$\operatorname{As}\left(u_{\tau}-u_{h \tau}\right)\left(t_{n}\right)=u^{n}-u_{h}^{n}=e^{n}$ and

$$
\left|\nabla_{h}\left(u_{\tau}-u_{h \tau}\right)(s)\right| \leq\left|\nabla_{h} e^{p-1}\right|+\left|\nabla_{h} e^{p}\right|, \forall s \in\left[t_{p-1}, t_{p}\right],
$$

the above estimate may be transformed into

$$
E\left(t_{n}\right)^{2} \lesssim \sum_{p=1}^{n}\left(\eta_{t}^{p}\right)^{2}+\left\|e^{n}\right\|^{2}+\sum_{p=0}^{n} \tau_{p}\left\|\nabla_{h} e^{p}\right\|^{2}+\left\|\partial_{t}\left(u_{\tau}-u_{h \tau}\right)\right\|_{L^{2}\left(0, t_{n} ; H^{-1}(\Omega)\right)}^{2}+\left\|f-\pi_{\tau} f\right\|_{L^{2}\left(0, t_{n} ; H^{-1}(\Omega)\right)}^{2} .
$$

We conclude using Theorem 5.2 and Corollary 5.3.

We now pass to the lower error bound. Summing the square of (40) on $p=1, \cdots, n$, we get

$$
\begin{aligned}
\sum_{p=1}^{n}\left(\eta_{t}^{p}\right)^{2} \lesssim & \int_{0}^{t_{n}}\left\|\nabla_{h} e_{\tau}(s)\right\|^{2} \mathrm{~d} s+\left\|\partial_{t} e_{\tau}\right\|_{L^{2}\left(0, t_{n} ; H^{-1}(\Omega)\right)}^{2} \\
& +\sum_{p=0}^{n} \tau_{p}\left(\left\|\nabla_{h}\left(u^{p}-u_{h}^{p}\right)\right\|^{2}+\left\|\nabla_{h}\left(u_{h}^{p-1}-u_{h}^{p-1}\right)\right\|^{2}\right)+\left\|f-\pi_{\tau} f\right\|_{L^{2}\left(0, t_{n} ; H^{-1}(\Omega)\right)}^{2} .
\end{aligned}
$$

By the estimate (37), we obtain

$$
\sum_{p=1}^{n}\left(\eta_{t}^{p}\right)^{2} \lesssim E\left(t_{n}\right)^{2}+\left\|f-\pi_{\tau} f\right\|_{L^{2}\left(0, t_{n} ; H^{-1}(\Omega)\right)}^{2}
$$


On the other hand, by Corollary 5.7, we have

$$
\sum_{p=1}^{n} \tau_{p}\left(\eta^{p}\right)^{2} \lesssim\left\|\partial_{t}\left(u_{\tau}-u_{h \tau}\right)\right\|_{L^{2}\left(0, t_{n} ; H^{-1}(\Omega)\right)}^{2}+\sum_{p=1}^{n} \tau_{p}\left(\left\|\nabla_{h}\left(u^{p}-u_{h}^{p}\right)\right\|^{2}+\left(\xi^{p}\right)^{2}\right)
$$

Again thanks to (37), we obtain

$$
\sum_{p=1}^{n} \tau_{p}\left(\eta^{p}\right)^{2} \lesssim E\left(t_{n}\right)^{2}+\sum_{p=1}^{n} \tau_{p}\left(\xi^{p}\right)^{2}
$$

The estimate (58) directly follows from (59) and (60).

Remark 6.2. If we assume that

$$
h_{p}^{2} \lesssim \tau_{p}, \forall 1 \leq p \leq N,
$$

then Theorem 6.1 states that the error $E\left(t_{n}\right)$ is equivalent to the global error estimator

$$
\left(\sum_{p=1}^{n}\left(\left(\eta_{t}^{p}\right)^{2}+\tau_{p}\left(\eta^{p}\right)^{2}\right)\right)^{1 / 2}
$$

up to approximation terms. Therefore this global error estimator may be used for an adaptive algorithm that has to respect (61).

\section{NUMERICAL EXPERIMENTS}

The following experiments will confirm our theoretical analysis. Since our main contribution concerns the spatial error estimator, we only concentrate our efforts to its validity. The first example is used to confirm the efficiency and reliability of our spatial error estimator. The second example illustrate the use of our spatial estimator by presenting a spatial adaptive algorithm for a solution having a singular behaviour in space.

\subsection{Test 1}

This example consists in solving the two dimensional heat equation on the unit square $\Omega=] 0,1[\times] 0,1[$. Here, we first use the Crouzeix-Raviart element on uniform meshes $T_{p h}=T_{h}$ obtained by dividing each segment by $n$ subintervals and dividing each obtained rectangle into two triangles (see Fig. 2).

The tests are performed with $T=1 s$ and the following exact solution $u$ :

$$
\left.u(x, y, t)=e^{-t} x y(x-1)(y-1) \text { in } \Omega \times\right] 0,1[,
$$

so that $u_{0}(x, y)=x y(x-1)(y-1)$ in $\Omega$ and $u(., t)_{\mid \Gamma}=0$, for all $\left.t \in\right] 0,1\left[\right.$. We fix $\tau_{p}=0.1 s$, then $N=T / \tau_{p}=10$. All numerical results will be presented at the final time $T=1 s(N=10)$.

First, we check that the numerical solution $u_{h}^{N}$ converges towards the exact one. For that purpose, in Figure 3, we have plotted $\left\|\nabla_{h} e^{N}\right\|$ as a function of the degrees of freedom $\left(D o F=3 n^{2}-4 n+2\right.$ with $\left.h=1 / n\right)$. A double logarithmic scale was used such that the slope of the curves yields the order of convergence. As we can see, this figure underlines the theoretical predicted optimal order of convergence $h$ (see [10]).

Now we investigate the main theoretical results which are the upper and lower error bounds (41) and (51). 


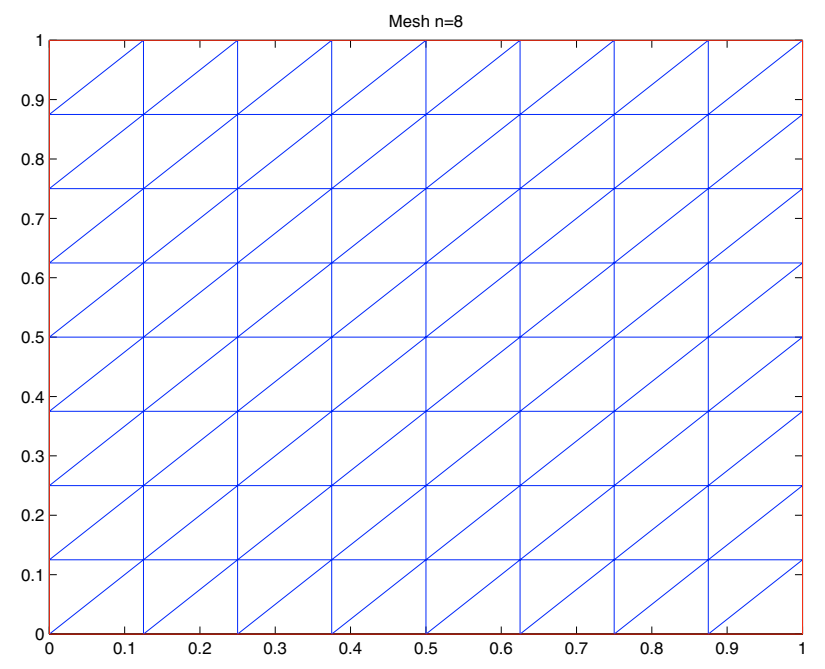

FIgURE 2. The uniform mesh on the unit square with $n=8$.

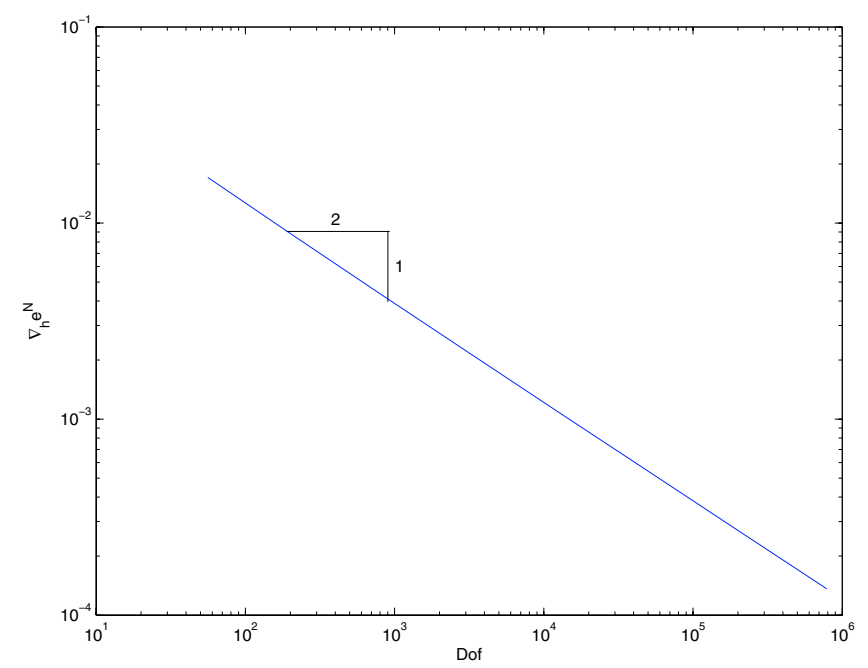

Figure 3. $\left\|\nabla_{h} e^{N}\right\|$ as a function of DoF for uniform meshes.

\subsubsection{Reliability of the spatial estimator}

First, we define the ratio of the left-hand side and the right-hand side of the inequality (41) at the last time $T=1 s:$

$$
q_{u p}^{N}=\frac{\left\|e^{N}\right\|^{2}+\sum_{p=1}^{N} \tau_{p}\left\|\nabla_{h} e^{p}\right\|^{2}}{\left\|e^{0}\right\|^{2}+\sum_{p=1}^{N} \tau_{p} \sum_{K \in T_{p h}}\left(\left(\eta_{K}^{p}\right)^{2}+h_{K}^{2}\left\|f^{p}-f_{h}^{p}\right\|_{K}^{2}\right)} .
$$




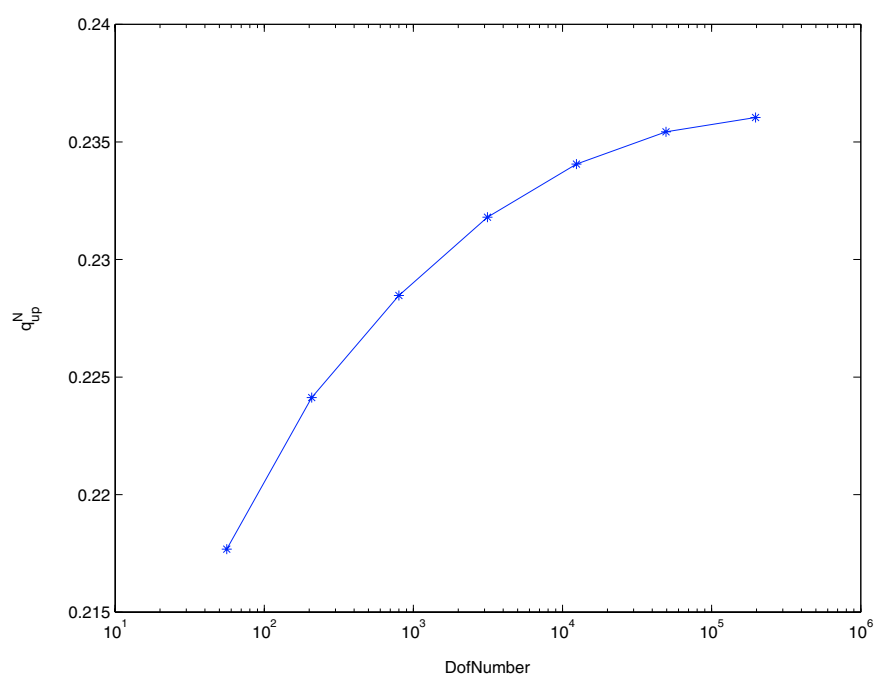

Figure 4. $q_{u p}^{N}$ wrt DoF for uniform meshes.

TABlE 1. $q_{u p}^{N}$ and $q_{l o w}^{N}$ wrt DoF for uniform meshes.

\begin{tabular}{||r|r|c|c||}
\hline $\boldsymbol{n}$ & $\boldsymbol{D o F}$ & $\boldsymbol{q}_{\mathbf{u p}}^{\mathbf{N}}$ & $\boldsymbol{q}_{\mathbf{l o w}}^{\mathbf{N}}$ \\
\hline \hline 4 & 56 & 0.21768 & 2.0782 \\
\hline 8 & 208 & 0.22413 & 2.5714 \\
\hline 16 & 800 & 0.22847 & 2.9010 \\
\hline 32 & 3136 & 0.23180 & 3.1265 \\
\hline 64 & 12416 & 0.23406 & 3.2208 \\
\hline 128 & 49408 & 0.23543 & 3.2843 \\
\hline 256 & 197120 & 0.23604 & 3.2930 \\
\hline 512 & 787456 & 0.23617 & 3.2975 \\
\hline
\end{tabular}

$q_{u p}^{N}$ is referred as the effectivity index. It measures the reliability of the estimator and is related to the global upper error bound. From Theorem 5.2, the ratio $q_{u p}^{N}$ is bounded from above. This can be confirmed by our numerical results presented in Figure 4 and Table 1. Hence, the spatial estimator is reliable.

\subsubsection{Efficiency of the spatial estimator}

Now, we define the (larger) ratio of the left-hand side and the right-hand side of the inequality (51) at the final time $T=1 s$ :

$$
q_{\text {low }}^{N}=\max _{K \in T_{p h}} \frac{\eta_{K}^{N}}{h_{K}\left\|\frac{e^{N}-e^{N-1}}{\tau_{p}}\right\|_{\omega_{K}}+\left\|\nabla_{h} e^{N}\right\|_{\omega_{K}}+h_{K}\left\|f^{N}-f_{h}^{N}\right\|_{\omega_{K}}} .
$$

$q_{\text {low }}^{N}$ is related to the local lower error bound and measures the efficiency of the estimator. According to Figure 5 (see also Tab. 1), $q_{\text {low }}^{N}$ is bounded from above as theoretically predicted in Theorem 5.6. Therefore our spatial estimator is also efficient. 


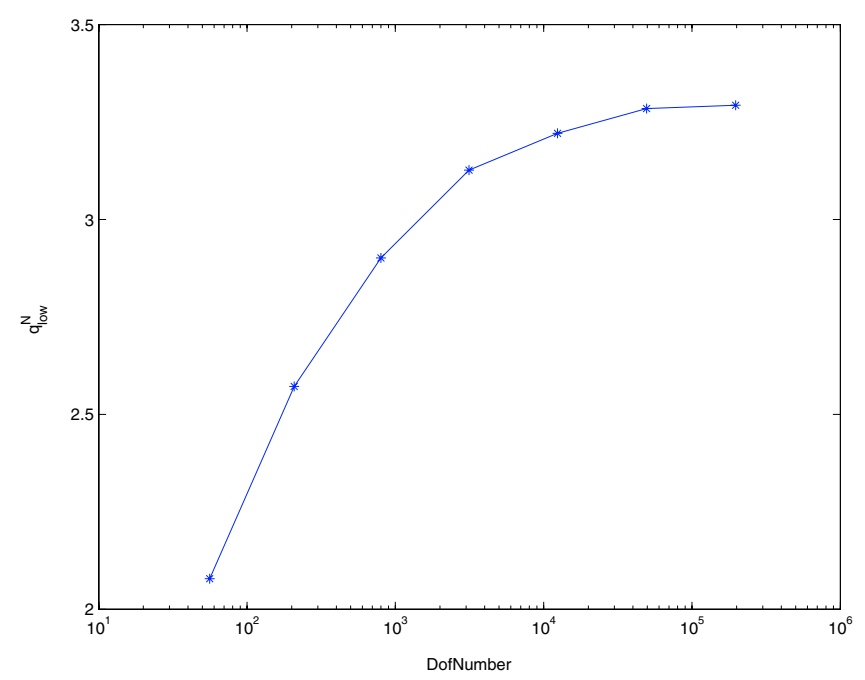

Figure 5. $q_{\text {low }}^{N}$ wrt DoF for uniform meshes.

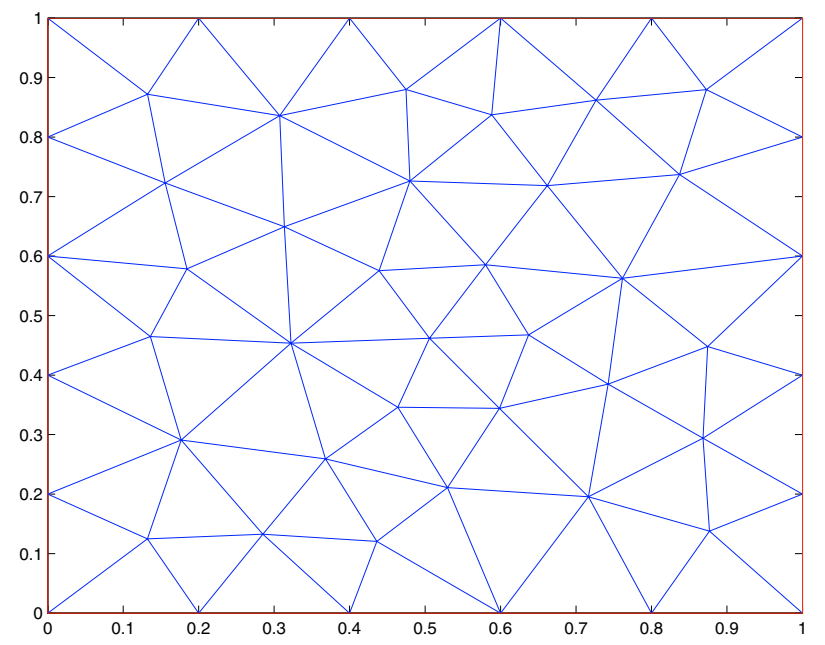

FiguRE 6 . The non structured mesh on the unit square with $h=0.2$.

\subsubsection{Non structured meshes}

In order to validate the reliability and efficiency of our spatial error estimator, we have approximated the same problem as before with the same elements but on different non structured meshes obtained by starting from a rough non structured mesh of size 0.2 (see Fig. 6) and by dividing each triangle into 4 triangles by the standard regular refinements [20]. Figures 7 and 8 (see also Tab. 2) show respectively the ratios $q_{u p}^{N}$ and $q_{l o w}^{N}$ with respect to the degrees of freedom. Again we may conclude that both ratios are bounded from above and consequently our spatial error estimator is reliable and efficient.

\subsection{Dependence of the error}

From our previous considerations, the error between the exact solution and its approximated one is expected to depend on the space and/or time discretization. In order to illustrate this phenomenon, as in [17], we exhibit 


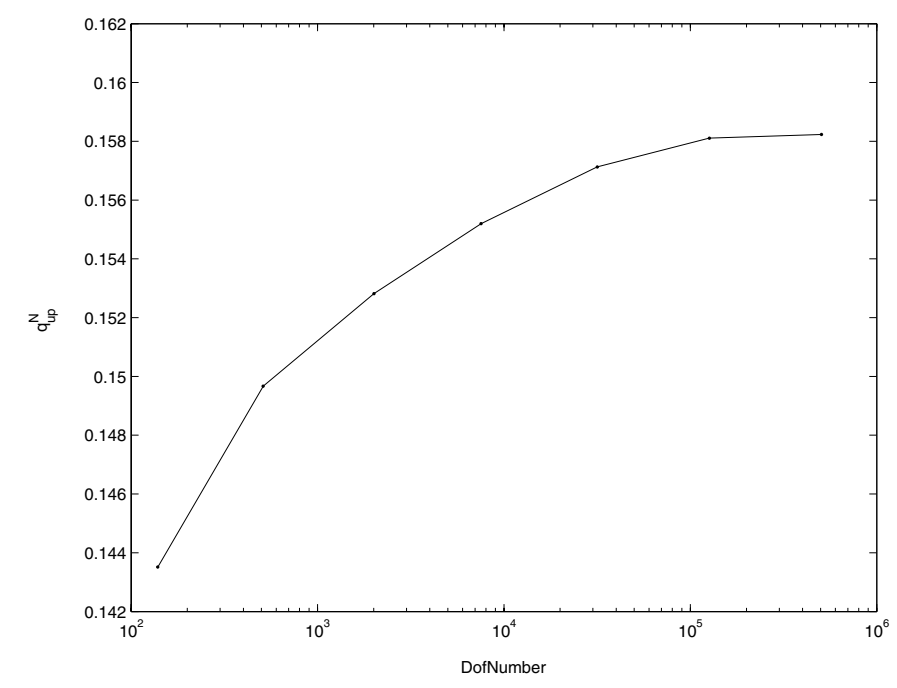

Figure 7. $q_{u p}^{N}$ wrt DoF for the non structured meshes.

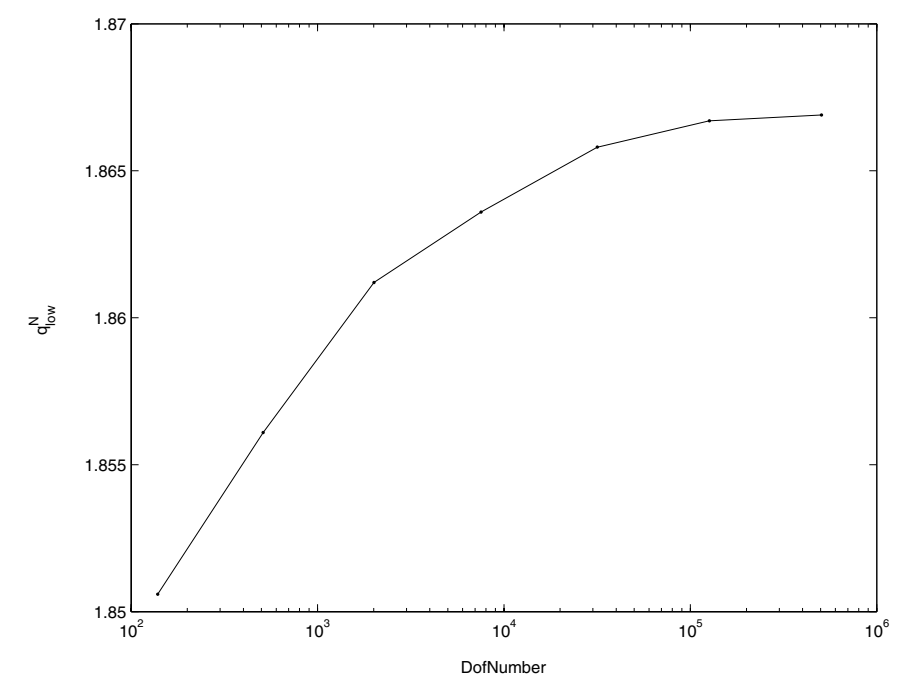

FiguRE 8. $q_{\text {low }}^{N}$ wrt DoF for the non structured meshes.

an example where the error due to the time discretization is more important than the error due to the space discretization, and another example where the converse phenomenon appears. For that purpose we consider the problem (1) for $\Omega=] 0,1[\times] 0,1\left[\right.$ and $T=1 s$, with the exact solutions $u_{1}$ and $u_{2}$ defined by:

$$
u_{1}(x, y, t)=\sin (10 \pi t / 2) \sin (\pi x / 2) \sin (\pi y / 2)
$$

and

$$
u_{2}(x, y, t)=\sin (10 \pi t / 2) \sin (10 \pi x / 2) \sin (10 \pi y / 2) .
$$

The numerical results are shown in Tables 3 and 4 , where we present the values of the space indicator $\eta$, the time indicator $\eta_{t}$, the error $\|e\|:=\left(\sum_{p=1}^{N} \tau_{p}\left\|\nabla_{h} e^{p}\right\|^{2}\right)^{1 / 2}$ and the spatial effectivity index $q_{u p}^{N}$ for different uniform triangulations and constant time steps. In the first case, we can conclude that the error is mainly due to the 
TABLE 2. $q_{u p}^{N}$ and $q_{l o w}^{N}$ wrt DoF for the non structured meshes.

\begin{tabular}{||r|r|c|c||}
\hline $\boldsymbol{h}$ & $\boldsymbol{D o F}$ & $\boldsymbol{q}_{\text {up }}^{\mathbf{N}}$ & $\boldsymbol{q}_{\text {low }}^{\mathbf{N}}$ \\
\hline \hline 0.2 & 139 & 0.14351 & 1.8506 \\
\hline 0.1 & 512 & 0.14967 & 1.8561 \\
\hline 0.05 & 2008 & 0.15282 & 1.8612 \\
\hline 0.025 & 7952 & 0.15520 & 1.8636 \\
\hline 0.0125 & 31648 & 0.15713 & 1.8658 \\
\hline 0.00625 & 126272 & 0.15811 & 1.8667 \\
\hline 0.003125 & 504448 & 0.15823 & 1.8669 \\
\hline
\end{tabular}

TABLE 3. Convergence results when using uniform triangulations and constant time steps for the first example.

\begin{tabular}{||l|l|l|l|l|l||l|l|l|l|l|l|}
\hline$h=1 / n$ & $\mathrm{~d} t$ & $\eta$ & $\eta_{t}$ & $\|e\|$ & $q_{u p}^{N}$ & $h=1 / n$ & $\mathrm{~d} t$ & $\eta$ & $\eta_{t}$ & $\|e\|$ & $q_{u p}^{N}$ \\
\hline \hline 0.1 & 0.1 & 0.096 & 0.65 & 0.31 & 3.6 & 0.1 & 0.05 & 0.062 & 0.34 & 0.19 & 3.3 \\
\hline 0.05 & 0.1 & 0.051 & 0.65 & 0.31 & 3.6 & 0.05 & 0.05 & 0.031 & 0.34 & 0.19 & 3.3 \\
\hline 0.025 & 0.1 & 0.025 & 0.65 & 0.31 & 3.6 & 0.025 & 0.05 & 0.016 & 0.34 & 0.19 & 3.3 \\
\hline 0.0125 & 0.1 & 0.012 & 0.65 & 0.30 & 3.7 & 0.0125 & 0.05 & 0.008 & 0.34 & 0.19 & 3.3 \\
\hline \hline 0.1 & 0.025 & 0.043 & 0.18 & 0.11 & 3.0 & 0.1 & 0.0125 & 0.041 & 0.09 & 0.06 & 2.7 \\
\hline 0.05 & 0.025 & 0.022 & 0.18 & 0.10 & 3.1 & 0.05 & 0.0125 & 0.021 & 0.09 & 0.06 & 2.7 \\
\hline 0.025 & 0.025 & 0.011 & 0.18 & 0.10 & 3.1 & 0.025 & 0.0125 & 0.010 & 0.09 & 0.05 & 2.8 \\
\hline 0.0125 & 0.025 & 0.005 & 0.18 & 0.10 & 3.1 & 0.0125 & 0.0125 & 0.005 & 0.09 & 0.05 & 2.8 \\
\hline
\end{tabular}

TABLE 4. Convergence results when using uniform triangulations and constant time steps for the second example.

\begin{tabular}{||l|l|l|l|l|l||l|l|l|l|l|l|}
\hline$h=1 / n$ & $\mathrm{~d} t$ & $\eta$ & $\eta_{t}$ & $\|e\|$ & $q_{u p}^{N}$ & $h=1 / n$ & $\mathrm{~d} t$ & $\eta$ & $\eta_{t}$ & $\|e\|$ & $q_{u p}^{N}$ \\
\hline \hline 0.1 & 0.1 & 4.8 & 7.5 & 5.2 & 2.9 & 0.1 & 0.05 & 4.8 & 3.9 & 4.9 & 1.4 \\
\hline 0.05 & 0.1 & 2.6 & 7.2 & 2.9 & 5.8 & 0.05 & 0.05 & 2.6 & 3.8 & 2.5 & 2.9 \\
\hline 0.025 & 0.1 & 1.3 & 7.2 & 2.1 & 7.9 & 0.025 & 0.05 & 1.3 & 3.8 & 1.4 & 7.9 \\
\hline 0.0125 & 0.1 & 0.65 & 7.1 & 1.6 & 8.4 & 0.0125 & 0.05 & 0.65 & 3.8 & 0.83 & 8.1 \\
\hline \hline 0.1 & 0.025 & 4.8 & 1.9 & 5.4 & 0.7 & 0.1 & 0.0125 & 4.8 & 1.0 & 5.4 & 0.5 \\
\hline 0.05 & 0.025 & 2.6 & 1.9 & 2.7 & 1.5 & 0.05 & 0.0125 & 2.6 & 1.0 & 2.7 & 0.8 \\
\hline 0.025 & 0.025 & 1.3 & 1.9 & 1.3 & 2.8 & 0.025 & 0.0125 & 1.3 & 1.0 & 1.3 & 1.3 \\
\hline 0.0125 & 0.025 & 0.65 & 1.9 & 0.69 & 5.5 & 0.0125 & 0.0125 & 0.65 & 1.0 & 0.68 & 2.8 \\
\hline
\end{tabular}

time discretization. Indeed from Table 3, we see that for a fixed time step and decreasing mesh sizes, the error is almost constant; while for a fixed mesh size and decreasing time steps, the error decreases. We moreover remark a close relationship between the error and the time indicator. For the second example, the error is mainly due to the time discretization, since we see converse relations between the error and the time steps 
and mesh sizes; while we clearly detect a relationship between the error and the space indicator. For the first example $q_{u p}^{N}$ is correlated to the error, while for the second one, the distorsion comes for the approximation terms. Let us further remark that the numerical experiments bring to light that the indicator $\eta_{t}$ is independent of $h$, while the indicator $\eta$ is mainly independent of $\tau_{p}$. This very important property of uncoupling the two error parts is effectively used in our adaptive algorithm described below, since the time (resp. space) refinements or unrefinements are (mainly) based on $\eta_{t}$ (resp. $\eta$ ).

\subsection{An adaptive algorithm}

From our theoretical considerations and the examples of the previous subsection, an adaptive algorithm has to use appropriately the space indicator $\eta$, the time indicator $\eta_{t}$ and the approximation error $\xi$. To design this algorithm, we first define the global indicator $\bar{\eta}$ as follows:

$$
\bar{\eta}:=\left(\sum_{n=1}^{N}\left(\left(\eta_{t}^{n}\right)^{2}+\tau_{n}\left(\eta^{n}\right)^{2}+\tau_{n}\left(\xi^{n}\right)^{2}\right)\right)^{1 / 2} .
$$

For our approximated solution $u_{h \tau}$, we define a relative error estimator Ind by:

$$
\text { Ind }^{2}=\frac{\bar{\eta}}{\int_{0}^{T}\left\|\nabla u_{h \tau}(\cdot, t)\right\|^{2} \mathrm{~d} t} .
$$

Let a preset tolerance $\delta$ and a parameter $0<\alpha<1$ be given. The goal of our adaptive scheme is to generate a sequence of sub-intervals $\left[t_{n-1}, t_{n}\right]$ and mesh triangulations $T_{n h}, n=1, \ldots, N$ such that Ind, defined by (62), is close to the preset of tolerance $\delta$, in the sense that

$$
(1-\alpha) \delta \leq \text { Ind } \leq(1+\alpha) \delta .
$$

To achieve these bounds, for all $n=1, \cdots, N$, we define two local bounds: a left one $\mathbf{L b}^{n}$ defined by

$$
\mathbf{L b}^{n}:=(1-\alpha)^{2} \delta^{2} \int_{t_{n-1}}^{t_{n}}\left\|\nabla u_{h \tau}(\cdot, t)\right\|^{2} \mathrm{~d} t
$$

and a right one $\mathbf{R b}^{n}$ defined by

$$
\mathbf{R b}^{n}:=(1+\alpha)^{2} \delta^{2} \int_{t_{n-1}}^{t_{n}}\left\|\nabla u_{h \tau}(\cdot, t)\right\|^{2} \mathrm{~d} t
$$

If, for all $n=1, \cdots, N$, the conditions

$$
\mathbf{L} \mathbf{b}^{n} \leq\left(\eta_{t}^{n}\right)^{2}+\tau_{n}\left(\eta^{n}\right)^{2}+\tau_{n}\left(\xi^{n}\right)^{2} \leq \mathbf{R} \mathbf{b}^{n}
$$

are satisfied, then summing from $n=1$ to $n=N$, we obtain (63). Thus our algorithm consists in finding time steps and triangulations such that (66) holds for all $n$. This will be achieved by using the elements $\eta^{n}$ and $\xi^{n}$ to control the mesh sizes, and using $\xi^{n}$ and $\eta_{t}^{n}$ to control the time steps. This adaptive algorithm is presented in Table 5. Note that it is similar to the one proposed in [17].

In order to test our adaptive scheme, we consider two relevant examples. The first one when the heat equation (1) is considered in the unit square $] 0,1[\times] 0,1[$ with the exact solution defined by (see [17])

$$
u(x, y, t)=\beta(t) * \exp \left(-50 * r^{2}(x, y, t)\right),
$$


TABLE 5. The adaptive algorithm.

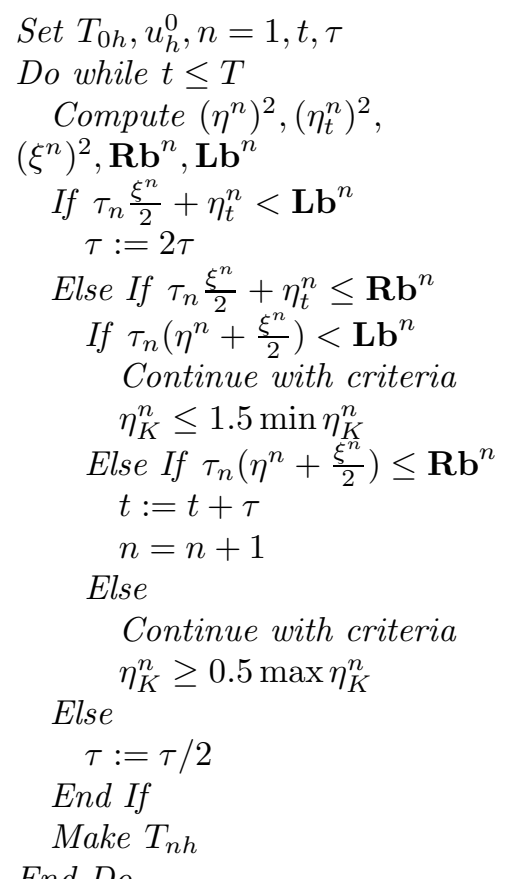

Initializations

Current time step is to small

Same time iteration with bigger step

Triangulation is too fine

Mesh Triangulation is correct

Incrementation of the current time step

Mesh Triangulation is too coarse

Same time with finer triangulation

Time step is too large

Same time iteration with smaller time step

Generate the new triangulation

End Do

TABLE 6. Some results for the Gaussian function with $\alpha=0.5$.

\begin{tabular}{||l|r|r|r||}
\hline Tolerance $\delta$ & Time Steps & Ind & $q_{\mathrm{up}}^{\mathbf{N}}$ \\
\hline \hline 1.0 & 10 & 0.62 & 1.4 \\
\hline 0.5 & 20 & 0.33 & 1.3 \\
\hline 0.25 & 40 & 0.17 & 1.2 \\
\hline 0.125 & 80 & 0.084 & 1.2 \\
\hline 0.0625 & 160 & 0.042 & 1.2 \\
\hline
\end{tabular}

with $r^{2}(x, y, t)=(x-0.4 * t-0.3)^{2}+(y-0.4 * t-0.3)^{2}$, and

$$
\left\{\begin{array}{l}
\beta(t)=1-\exp \left(-50 *(0.98 * t+0.01)^{2}\right) \quad \text { if } t<0.5 \\
\beta(t)=1-\exp \left(-50 *(1-0.98 * t+0.01)^{2}\right) \quad \text { else. }
\end{array}\right.
$$

This means that $u$ is a Gaussian function which center moves from point $(0.3,0.3)$ at time $t=0 \mathrm{~s}$ to point $(0.7,0.7)$ at time $t=1 \mathrm{~s}$.

The obtained meshes at times 0.1, 0.5 and 1 are shown in Figures 9 to 11 respectively with the tolerance $\delta=0.25$ and the parameter $\alpha=0.5$. From these figures we may conclude that the meshes are refined in the region of a large gradient of the solution and then follow correctly the moving centers. Moreover from Table 6, we see for different tolerance parameters, that the effectivity index is quite close to 1 .

As second example, we consider the heat equation (1) in the L-shape domain ] $-1,1\left[{ }^{2} \backslash\right] 0,1[\times] 0,-1[$ with exact solution defined by

$$
u(r, \theta)=\mathrm{e}^{-t} * r^{2 / 3} \sin \left(\frac{2}{3} \theta\right),
$$




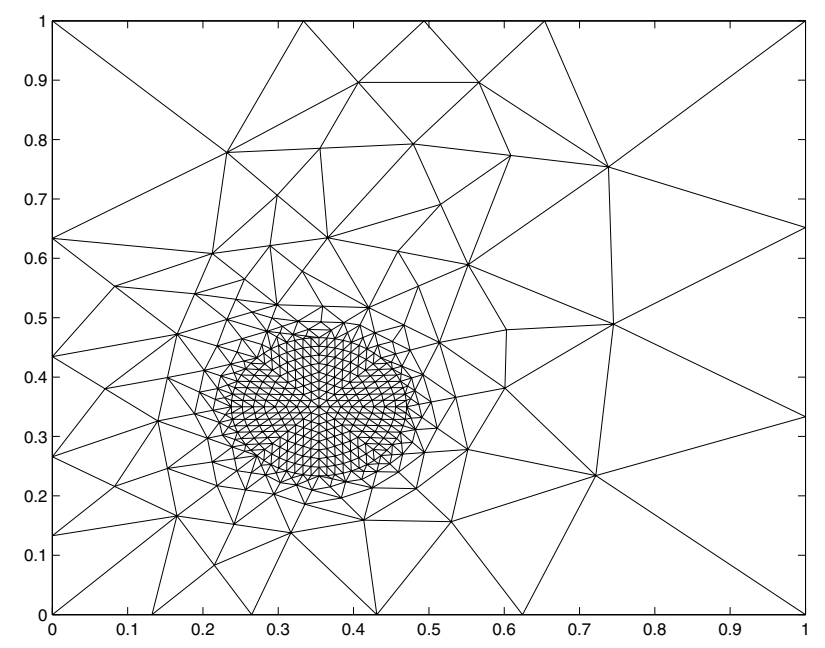

FIGURE 9. $n=4, t_{n}=0.1 s, N v=442$.

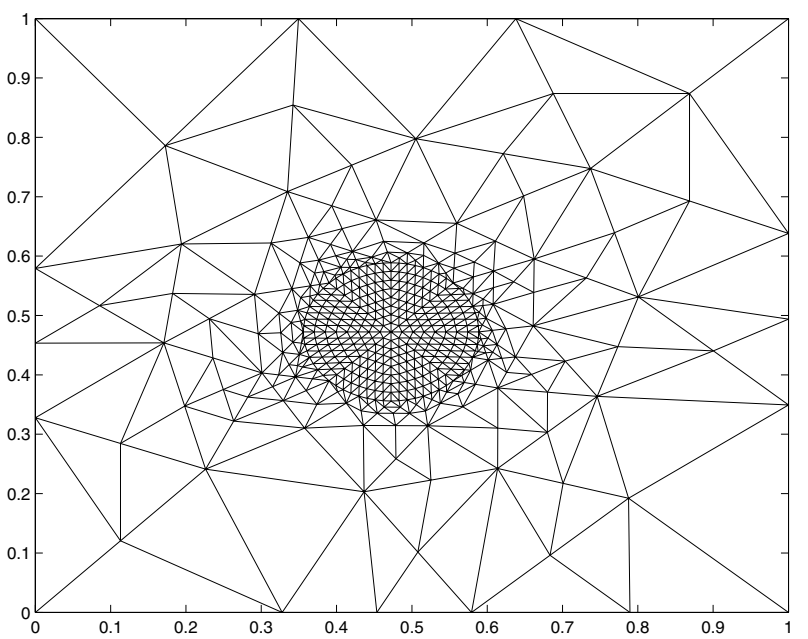

FiguRE 10. $n=20 t_{n}=0.5 s, N v=462$.

where $(r, \theta)$ are polar coordinates centred at $(0,0)$. In that case, $u$ has a singular behaviour along the edge $(0,0) \times] 0, T[$.

Figures 12 to 14 present the obtained meshes at times $0.1,0.5$ and 1 , respectively with the tolerance $\delta=0.25$ and the parameter $\alpha=0.5$. As expected the meshes are refined near the singular point, namely the origin. As previously Table 7 confirms a good effectivity index for different tolerance parameters.

\section{Conclusion}

We have proposed and analysed an a posteriori error estimator for the heat equation. Our investigations cover the nonconforming finite element discretization (Crouzeix-Raviart) on 2D and 3D domains. Much effort has been taken to prove the global upper and lower bound errors under quite realistic conditions. The main theoretical results, which are the upper and the lower spatial error bounds, are confirmed experimentally. More precisely the values $q_{u p}^{N}$ and $q_{l o w}^{N}$ are bounded from above as other problem classes $(c f$. [14, 18]). Finally a 


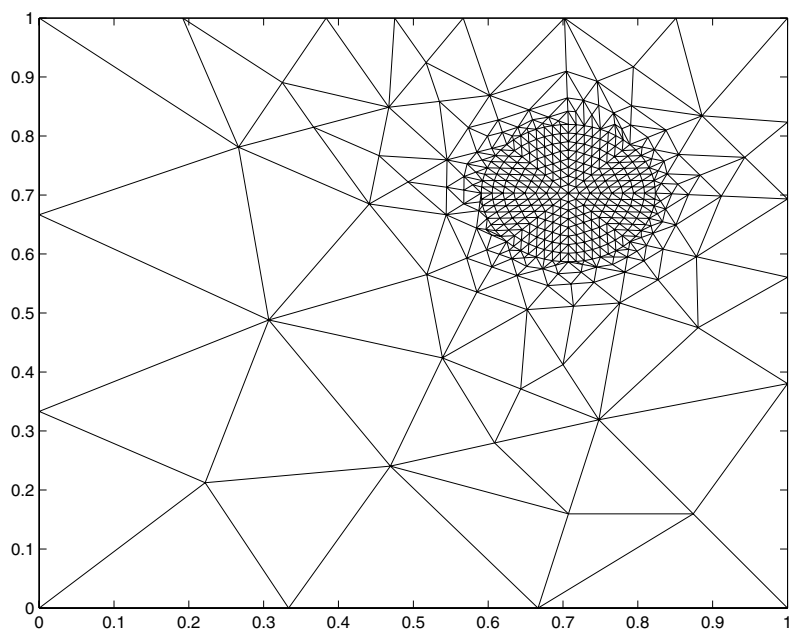

FiguRE 11. $n=40 t_{n}=1 s, N v=470$.

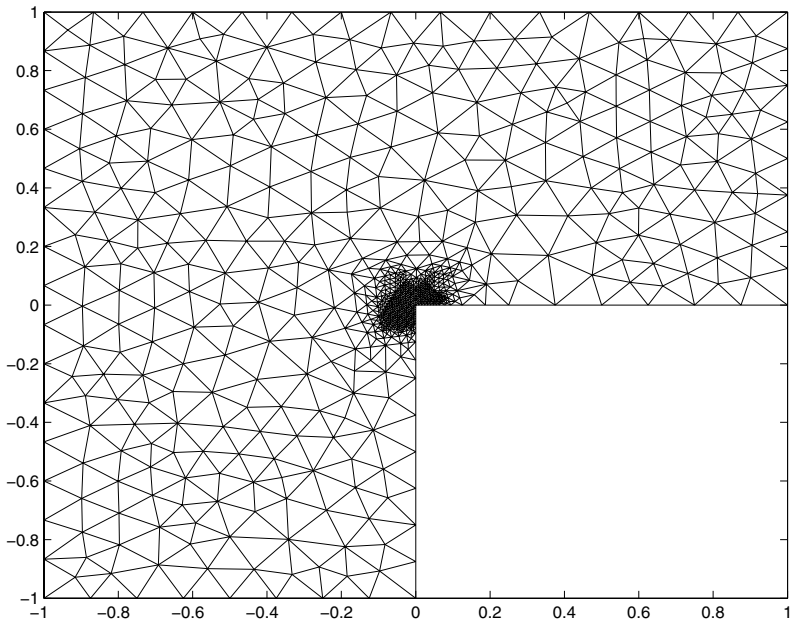

Figure 12. $n=4, t_{n}=0.1 s, N v=836$.

TABLE 7. Some results for the singular function with $\alpha=0.5$.

\begin{tabular}{||l|r|r|l||}
\hline Tolerance $\delta$ & Time Steps & Ind & $q_{\mathbf{u p}}^{\mathbf{N}}$ \\
\hline \hline 1.0 & 10 & 0.88 & 1.8 \\
\hline 0.5 & 20 & 0.42 & 1.6 \\
\hline 0.25 & 40 & 0.23 & 1.6 \\
\hline 0.125 & 80 & 0.11 & 1.6 \\
\hline 0.0625 & 160 & 0.055 & 1.5 \\
\hline
\end{tabular}




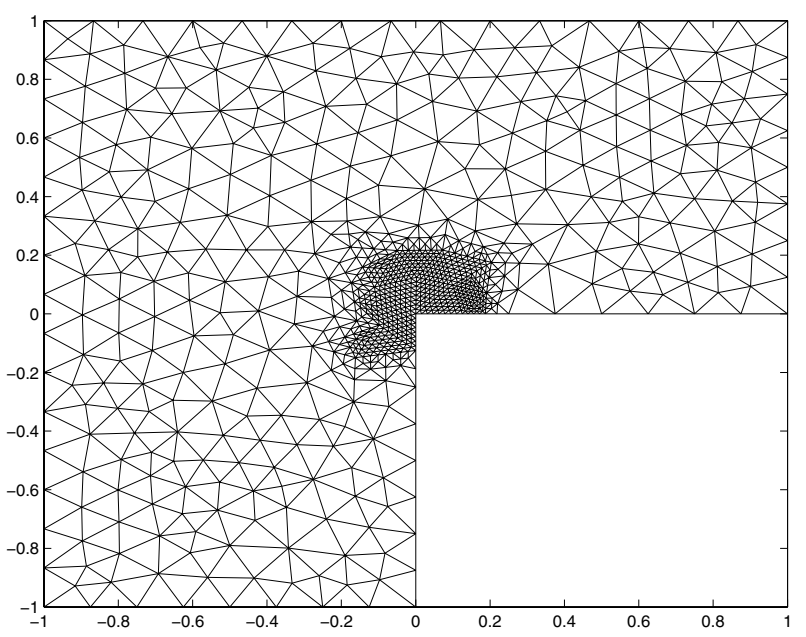

FiguRE 13. $n=20 t_{n}=0.5 s, N v=872$.

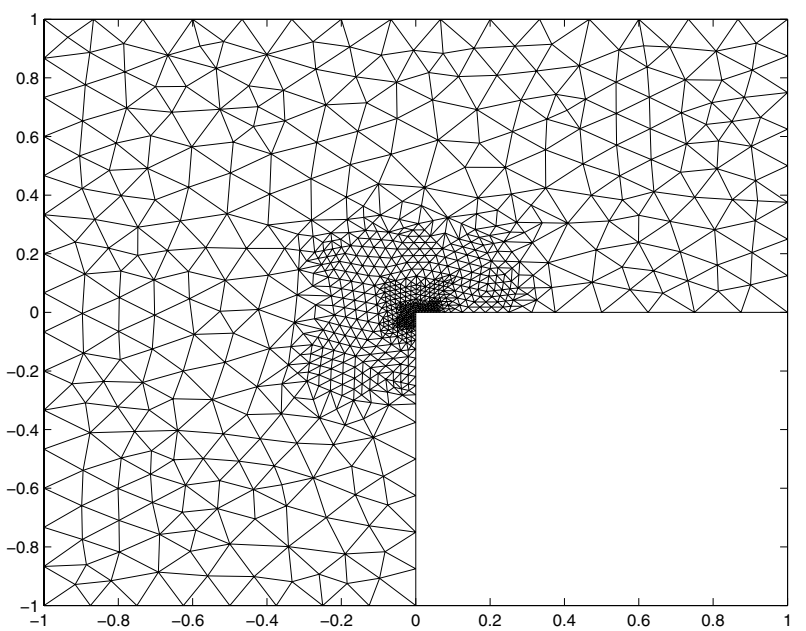

FIGURE 14. $n=40 t_{n}=1 s, N v=874$.

space-time adaptive algorithm based on our error estimator is proposed and tested on two relevant examples. In both cases, the obtained meshes follows the singularity of the solution, which confirms the validity of our algorithm.

\section{REFERENCES}

[1] Y. Achdou, C. Bernardi and F. Coquel, A priori and a posteriori error analysis of finite volume discretizations of Darcy's equations. Numer. Math. 96 (2003) 17-42.

[2] G. Acosta and R.G. Durán, The maximum angle condition for mixed and non-conforming elements, Application to the Stokes equations. SIAM J. Numer. Anal. 37 (1999) 18-36.

[3] T. Apel, Anisotropic finite elements: Local estimates and applications. Adv. Numer. Math. Teubner, Stuttgart (1999).

[4] T. Apel and S. Nicaise, The inf-sup condition for some low order elements on anisotropic meshes. Calcolo 41 (2004) 89-113.

[5] T. Apel, S. Nicaise and J. Schröberl, A non-conforming finite element method with anisotropic mesh grading for the stokes problem in domains with edges. IMA J. Numer. Anal. 21 (2001) 843-856. 
[6] A. Bergam, C. Bernardi and Z. Mghazli, A posteriori analysis of the finite element discretization of some parabolic problem. Preprint Laboratoire J.-L. Lions 01045, Université Paris 6 (2001).

[7] A. Bergam, C. Bernardi and Z. Mghazli, A posteriori analysis of the finite element discretization of a nonlinear parabolic equation. (2004) (to appear).

[8] C. Bernardi and B. Métivet, Indicateurs d'erreur pour l'équation de la chaleur. Rev. Européenne Élém. Finis 9 (2000) $425-438$.

[9] C. Bernardi and R. Verfürth, A posteriori error analysis of the fully discretized time-dependent Stokes equations. ESAIM: M2AN 38 (2004) 437-455.

[10] P. Brenner, M. Crouzeix and V. Thomée, Single step methods for inhomogeneous linear differential equations in banach space. RAIRO Anal. Numér. 16 (1982) 5-26.

[11] P. Ciarlet, The finite element method for elliptic problems. North Holland (1996).

[12] P. Clément, Approximation by finite element functions using local regularization. RAIRO Anal. Numér. 2 (1975) 77-84.

[13] E. Creusé, G. Kunert and S. Nicaise, A posteriori error estimation for the Stokes problem: Anisotropic and isotropic discretizations. Math. Models Methods Appl. Sci. 14 (2004) 1297-1341.

[14] E. Dari, R. Durán, C. Padra and V. Vampa, A posteriori error estimators for nonconforming finite element methods. RAIRO Modél. Math. Anal. Numér. 30 (1996) 385-400.

[15] V. Girault and P.-A. Raviart, Finite elements methods for Navier-Stokes equations, Theory and Algorithms. Springer Series in Computational Mathematics, Berlin (1986).

[16] C. Johnson, Y.-Y. Nie and V. Thomée, An a posteriori error estimate and adaptive timestep control for a backward Euler discretization of a parabolic problem. SIAM J. Numer. Anal. 27 (1990) 277-291.

[17] M. Picasso, Adaptive finite elements for a linear parabolic problem. Comput. Methods Appl. Mech. Engrg. 167 (1998) $223-237$.

[18] M. Picasso, An anisotropic error indicator based on Zienkiewicz-Zhu error estimator: Application to elliptic and parabolic problems. SIAM J. Sci. Comput. 24 (2003) 1328-1355.

[19] L.R. Scott and S. Zhang, Finite element interpolation of non-smooth functions satisfying boundary conditions. Math. Comp. 54 (1990) 483-493.

[20] R. Verfürth, A review of a posteriori error estimation and adaptive mesh-refinement techniques. Wiley-Teubner, Chichester, Stuttgart (1996).

[21] R. Verfürth, Error estimates for some quasi-interpolation operators. ESAIM: M2AN 33 (1999) 695-713.

[22] R. Verfürth, A posteriori error estimates for finite element discretization of the heat equation. Calcolo 40 (2003) 195-212.

To access this journal online:

www.edpsciences.org 